www. revistadyo.com

\title{
Métodos de planificación y secuenciación Heijunka inspirados en el problema del Reparto en Sistemas electorales
}

Joaquín Bautista-Valhondo

https://doi.org/10.37610/dyo.v0i73.590
Recibido: 07 de Julio de 2020

Aceptado: 30 de Septiembre de 2020

\section{Resumen}

El término Heijunka se asocia a la regularidad de la producción, siendo esta una propiedad deseable en todo plan de producción por facilitar la gestión de operaciones y propiciar la reducción de stocks. En este marco conceptual, se establecen tres propiedades deseables que deben cumplir, dentro de lo posible, los planes y las secuencias para ser considerados Heijunka. Estas son: (1) Cuota, (2) Homogeneidad y (3) Monotonía en producción.

Tras una introducción sobre el entorno productivo bajo estudio, se formula un programa matemático para obtener planes y secuencias regulares por cuotas de producción y se ofrece una heurística inspirada en el procedimiento de los Restos mayores del Problema del Reparto, la cual se aplica a un ejemplo sobre fabricación de motores. Se definen los conceptos periodicidad ideal de productos y secuenciación regular por fechas idóneas, quedando reflejados en un programa matemático cuyo propósito es determinar secuencias regulares, minimizando las desviaciones (absolutas, cuadráticas, etc.) entre fechas reales e ideales de fabricación.

También, se propone la familia de algoritmos Métodos de los Multiplicadores, aplicando algunos de ellos al conjunto de instancias Nissan-9Eng.I. Y se concluye con la idea de que dichos métodos son útiles para secuenciar productos bajo el ideario Heijunka, pues todos ellos verifican al menos dos de las tres propiedades deseables impuestas.

\section{Palabras clave}

Heijunka; Just in time; Métodos de los Divisores; Métodos de los Multiplicadores; Programación matemática; Problema del Reparto; Sistemas electorales.

\section{Introducción}

Entre principios y mediados del pasado siglo, muchas empresas de automoción producían un solo producto, sin variantes y en grandes cantidades. No obstante, en los años en que la empresa Ford había adquirido una hegemonía indiscutible en la industria del automóvil mediante su modelo T ya se producían en sus fábricas algunas variantes de este modelo (turismos, furgonetas, camiones de ligero tonelaje, etc.); de hecho, el propio modelo $\mathbf{T}$ iba evolucionando con el paso del tiempo. Pero, llegó el momento en que la hegemonía de Ford se derrumbó ante General Motors que tuvo la capacidad de ofrecer una amplia gama de marcas y modelos, y así satisfacer al consumidor en necesidades y apetencias.

\footnotetext{
$凶 \quad$ Joaquín Bautista-Valhondo * joaquin.bautista@upc.edu

iD ORCID: 0000-0002-2214-4991

* IOC ETSEIB Universitat Politècnica de Catalunya

Av. Diagonal 647, 08028, Barcelona, Spain.
}

El fenómeno de la amplia gama con oferta de opciones fue incorporado a la mayoría de industrias clásicas y servicios. Las empresas más hábiles fueron capaces de ganar cuota de mercado, generando nuevos productos y más variedad, mientras que las que no lo hicieron se vieron condenadas a desaparecer.

La necesidad de producir una amplia variedad de productos generó nuevos problemas de gestión. Dicha variedad propició la aparición de los sistemas de producción de modelos mixtos, en los que, por ejemplo, una línea de montaje se dedica a fabricar productos que, sin ser idénticos, se parecen entre sí. Empleamos el término variantes de un producto para denominar conceptualmente esta semblanza relativa entre ciertos bienes o servicios.

La fabricación de variantes de un producto en un mismo sistema productivo implica llevar a cabo tareas de preparación cada vez que se lanza la fabricación de una de ellas. Si los tiempos de preparación de una a otra variante son elevados, entonces, la fabricación se efectúa por lotes de magnitud significativa, lo que implica más stocks y poca flexibilidad para hacer frente a desviaciones de la demanda real frente a las previsiones. 
Consecuentemente, la apuesta por la variedad obliga a reducir los tiempos de preparación, convirtiéndose tal reducción en principal objetivo a la hora de diseñar el producto y el proceso mediante el estudio detallado de métodos de trabajo e instrumentos a utilizar para definir las tareas de lanzamiento de operaciones en el sistema productivo. Cuando los tiempos de preparación se reducen a valores poco significativos (v.gr. línea de montaje), se dice que se dispone de un sistema de producción flexible. Disponer de un sistema de este tipo es condición necesaria para afrontar la variedad, pero no suficiente, ya que la flexibilidad requiere, además, herramientas matemáticas que permitan obtener y evaluar con agilidad planes regulares de producción y secuencias regulares de modelos mixtos.

El término "regular" se emplea para expresar que el objeto referido presenta similitud o continuidad en su conjunto, desarrollo, distribución o duración. Para el presente trabajo, se proponen dos definiciones que vinculan la organización de la producción en contexto JIT (Just in Time) [1] con dicho término.

d1. Plan regular de producción mixta: Diremos que un plan de producción es regular cuando, teniendo en cuenta la demanda y las restricciones de capacidad del sistema productivo, las cantidades a fabricar de los tipos de producto son lo más parecidas posible en todos los períodos (días, semanas o meses) del horizonte de planificación.

d2. Secuencia regular de productos mixtos: Diremos que una secuencia es regular en producción cuando su concatenación repetida, el número de veces que sea necesario, permite construir un plan regular de producción mixta, preservando así el mix de producción en la medida de lo posible.

La regularidad de la producción se asocia al término japonés Heijunka (alisado de la producción), afectando a planes y secuencias de producción ([2], [3]), así como al cálculo del tamaño de los buffers entre procesos [4]. Esta propiedad es deseable en todo plan de producción, independientemente de su horizonte, pues facilita la dirección de operaciones y permite reducir algunas ineficiencias. Entre las diversas ventajas que ofrece el ideario Heijunka se encuentra las que siguen.
(1) Reducción de stocks, produciendo sólo lo necesario para satisfacer la demanda.

(2) Reducción de la capacidad productiva requerida, ajustando los equipos, maquinaria, instrumentos, materiales componentes y mano de obra.

(3) Reducción de plazos de entrega al proceso siguiente y desde procesos anteriores.

(4) Reducción del volumen de información para dirigir las operaciones productivas y logísticas, tanto a nivel interno como en lo concerniente a proveedores externos.

(5) Transparencia en la relación con proveedores (externos e internos), propiciando un esfuerzo uniforme a lo largo del tiempo para todos.

Los próximos apartados están orientados a ilustrar cómo aplicar algunos métodos del problema del Reparto a la planificación y secuenciación de modelos mixtos, cuando incorporamos la propiedad de regularidad de la producción.

\section{Planificación Heijunka con el método de los Restos Mayores}

Las primeras aportaciones formales del problema del Reparto se dieron en el campo de las Ciencias Sociales (ámbito político) con el reparto de escaños de una cámara de representantes ([5], [6], [7]), siendo el método de los Restos Mayores (RM) de Hamilton uno de los más relevantes. Para ilustrar la aplicación de tal método al problema de planificación Heijunka nos apoyaremos en el Ejemplo 1.

\section{Ejemplo 1:}

En la línea L1 de modelos mixtos se deben ensamblar, durante 8 turnos de trabajo, 1080 motores de 9 tipos (P1 a P9) clasificados en 3 familias: SUV, VAN y TRUCK (ver ejemplo en Figura 1). Las demandas planificadas por tipo de motor para un horizonte de 8 turnos se muestran en la Tabla 1. Considerando un contexto de fabricación JIT, se debe determinar un plan Heijunka preservando el mix de producción a lo largo del horizonte y manteniendo constante el volumen diario de producción.
Tabla 1 Demanda de motores por tipo con un horizonte de 8 turnos en la Línea L1.

\begin{tabular}{c|ccccccccc|c}
\hline Motor & P1 & P2 & P3 & P4 & P5 & P6 & P7 & P8 & P9 & Total \\
\hline Demanda $(8 \mathrm{~T})$ & 12 & 12 & 25 & 37 & 61 & 98 & 160 & 258 & 417 & $\mathbf{1 0 8 0}$ \\
\hline
\end{tabular}


Informalmente, el problema consiste en determinar 72 números enteros y colocarlos en una tabla, a modo de sudoku, de forma que por filas sumen las demandas de los productos de la Tabla 1, y por columnas sumen la capacidad de producción de un turno de trabajo igual a 135 motores; además, los valores por filas deben ser lo más parecidos posible.

Figura 1 Características producto-proceso: (i) 747 piezas y 330 referencias - (ii) 140 operaciones de montaje $\cdot$ (iii) 42 operarios para un turno de 135 motores • (iv) 9 tipos de motores de 3 familias: SUV (p1 a p3), furgonetas (p4, p5) y camiones MT (p6 a p9) • (v) Turno efectivo $6 \mathrm{~h}$ $45^{\prime}$ y ciclo de 3 ,

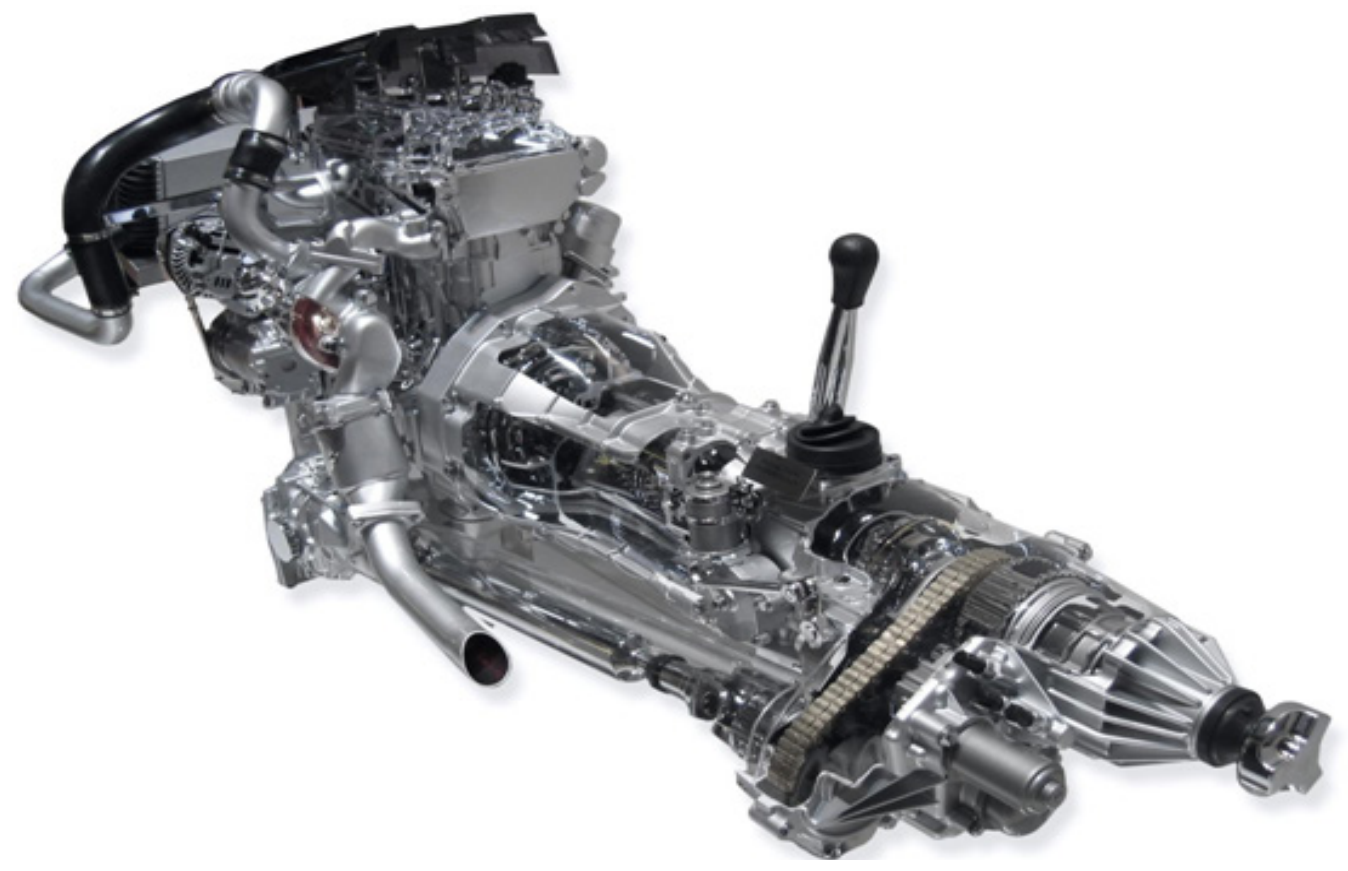

La vaguedad de la expresión "los valores por filas deben ser lo más parecidos posible" deja abierta la posibilidad de que exista más de una solución. Un plan Heijunka posible para el Ejemplo 1 es el que se muestra en la Tabla 2.
Tabla 2 Plan Heijunka-1: Producción de cada tipo de motor (P1 a P9) en cada turno $(\mathrm{t}=1, \ldots, 8)$.

\begin{tabular}{c|cccccccc|c}
\hline Plan H.1 & \multicolumn{7}{|c|}{ Plan de producción por turnos de trabajo: $x_{i, t}$} & \\
\hline Motor/Turno & 1 & 2 & 3 & 4 & 5 & 6 & 7 & 8 & Total \\
\hline P1 & 2 & 1 & 2 & 1 & 2 & 1 & 2 & 1 & 12 \\
P2 & 1 & 2 & 1 & 2 & 1 & 2 & 1 & 2 & 12 \\
P3 & 3 & 3 & 3 & 4 & 3 & 3 & 3 & 3 & 25 \\
P4 & 5 & 4 & 5 & 5 & 4 & 5 & 4 & 5 & 37 \\
P5 & 8 & 7 & 8 & 7 & 8 & 8 & 7 & 8 & 61 \\
P6 & 12 & 13 & 12 & 12 & 12 & 12 & 13 & 12 & 98 \\
P7 & 20 & 20 & 20 & 20 & 20 & 20 & 20 & 20 & 160 \\
P8 & 32 & 33 & 32 & 32 & 32 & 32 & 33 & 32 & 258 \\
P9 & 52 & 52 & 52 & 52 & 53 & 52 & 52 & 52 & 417 \\
\hline Total & 135 & 135 & 135 & 135 & 135 & 135 & 135 & 135 & $\mathbf{1 0 8 0}$ \\
\hline
\end{tabular}


Para hallar una solución del problema, se puede recurrir al tanteo por prueba y error en hoja de cálculo, ensayando con valores enteros en la Tabla 2 hasta conseguir que todos los números cuadren en el sudoku. Obviamente, esta no es la forma más cartesiana para resolver el problema, así que, después de algunas definiciones previas, utilizaremos la programación matemática para formalizar el problema que nos ocupa.
En primer lugar, a partir de las demandas de los 9 tipos de motor y el horizonte del plan, se determina la demanda por turno $d(d=1080 / 8=135)$, igual a la capacidad por turno, así como el mix de los 9 tipos de motor $\left(\lambda_{i}, \forall i=1 \ldots, 9\right)$. En la Tabla 3 , se muestran los valores de dicho mix de producción representados por el vector $\vec{\lambda}$.
Tabla 3 Plan Heijunka-1: Demanda y proporción de motores $\left(\mathrm{d}_{\mathrm{i}}, \lambda_{\mathrm{i}}\right)$ para 8 turnos en la Línea L1.

\begin{tabular}{c|ccccccccc|c}
\hline$i$ & P1 & P2 & P3 & P4 & P5 & P6 & P7 & P8 & P9 & Total \\
\hline$d_{i}$ & 12 & 12 & 25 & 37 & 61 & 98 & 160 & 258 & 417 & $\mathbf{1 0 8 0}$ \\
\hline$\lambda_{i}$ & 0,011 & 0,011 & 0,023 & 0,034 & 0,056 & 0,091 & 0,148 & 0,239 & 0,386 & $\mathbf{1}$ \\
\hline
\end{tabular}

Los valores del mix de producción representan las proporciones con las que los productos están presentes en el plan, y permiten determinar las producciones ideales acumuladas de cada tipo de motor hasta cada turno de fabricación. Por ejemplo, la proporción del motor P5 es $\lambda_{5}=d_{5} / D=61 / 1080=5,648 \%$, lo cual significa que, llegados al cuarto turno de trabajo $(\mathrm{t}=4)$, la producción ideal de dicho tipo, que llamaremos cuota de producción del motor $\mathrm{P} 5$, es $\mathrm{Q}_{5,4}=0,05648 \times 135 \times 4$, es decir: $\mathrm{Q}_{5,4}=30,50$ motores. En general, las cuotas de producción se determinarán según la fórmula (1).

$$
Q_{i, t}=\lambda_{i} \times d \times t \quad \forall i=1, \ldots, m ; \forall t=1, \ldots, T
$$

Donde:

I,i Conjunto de tipos de productos. Índices de tipo producto: $\mathrm{i}=1, . .,|\mathrm{I}|$.

m Número de tipos de producto del conjunto I: $\mathrm{m}=|\mathrm{I}|$.

$\mathrm{T}, \mathrm{t}$ Horizonte del plan de producción dividido en períodos (turno, día, mes). Índice del período: $\mathrm{t}=1, . ., \mathrm{T}$.

$\vec{d}, D, d \quad$ Vector plan de demanda $\vec{d}=\left(d_{1}, \ldots, d_{m}\right)$. Demanda total: $D=\sum_{\forall i} d_{i}$. Y demanda por período $d=D / T$, igual a la capacidad del sistema por período. $\vec{\lambda} \quad$ Vector mix de producción $\vec{\lambda}=\left(\lambda_{1}, \ldots, \lambda_{\mathrm{m}}\right): \vec{\lambda}=\overrightarrow{\mathrm{d}} / \mathrm{D}$

$\mathrm{Q}_{\mathrm{i}, \mathrm{t}} \quad$ Cuota de producción acumulada del producto $\mathrm{i} \in \mathrm{I}$ hasta el período $\mathrm{t}=1, \ldots, \mathrm{T}$.

En la Tabla 4 se muestran los valores de las cuotas de producción $\mathrm{Q}_{\mathrm{i}, \mathrm{t}}$, para todos los tipos de motor $(\mathrm{i}=1, \ldots, 9) \mathrm{y}$ hasta cada turno de trabajo $(\mathrm{t}=1, \ldots, 8)$.

Obviamente, la producción de motores debe ser un número natural, siendo $\mathrm{Q}_{5,4}=30,50$ un valor ideal orientativo que deberá convertirse en entero ajustándolo lo mejor posible al valor teórico proporcionado por la cuota $\mathrm{Q}_{54}$. Intuitivamente, podemos admitir que la producción real ajustada del motor tipo P5 hasta el $4^{\circ}$ turno debe ser 30 motores o bien 31 motores, según convenga.

En general, notaremos por $\mathrm{X}_{\mathrm{i}, \mathrm{t}}$ la producción real acumulada del producto tipo i $(\forall \mathrm{i})$ hasta el turno de fabricación $\mathrm{t}(\forall \mathrm{t})$, siendo la producción ajustada a su cuota $X_{i, t}{ }^{*}=\left\lfloor Q_{i, t}\right\rfloor$, o bien $\mathrm{X}_{\mathrm{i}, \mathrm{t}}{ }^{*}=\left[\mathrm{Q}_{\mathrm{i}, \mathrm{t}}\right\rceil$, donde $[\mathrm{Q}]$ representa el mayor entero inferior o igual a $Q$ y se llama cuota inferior, y [Q] es el menor entero superior o igual a Q y se llama cuota superior. Si se cumple: $\left[\mathrm{Q}_{\mathrm{i}, \mathrm{t}}\right\rfloor \leq \mathrm{X}_{\mathrm{i}, \mathrm{t}}{ }^{*} \leq\left\lceil\mathrm{Q}_{\mathrm{i}, \mathrm{t}}\right\rceil \forall \mathrm{i} \forall \mathrm{t}$, se dice que se satisface la Propiedad Cuota. 
Tabla 4 Plan Heijunka-1: Cuotas de producción acumulada en la Línea 1 para cada tipo de motor $(\mathrm{P} 1$ a P9) y para cada turno de trabajo $(\mathrm{t}=1, \ldots, 8)$.

\begin{tabular}{c|cccccccc}
\hline Plan H.1 & \multicolumn{7}{|c}{ Cuotas de producción acumula por turnos de trabajo: $Q_{i, t}$} \\
\hline Motor/Turno & 1 & 2 & 3 & 4 & 5 & 6 & 7 & 8 \\
\hline P1 & 1,50 & 3,00 & 4,50 & 6,00 & 7,50 & 9,00 & 10,50 & 12,00 \\
P2 & 1,50 & 3,00 & 4,50 & 6,00 & 7,50 & 9,00 & 10,50 & 12,00 \\
P3 & 3,13 & 6,25 & 9,38 & 12,50 & 15,63 & 18,75 & 21,88 & 25,00 \\
P4 & 4,63 & 9,25 & 13,88 & 18,50 & 23,13 & 27,75 & 32,38 & 37,00 \\
P5 & 7,63 & 15,25 & 22,88 & 30,50 & 38,13 & 45,75 & 53,38 & 61,00 \\
P6 & 12,25 & 24,50 & 36,75 & 49,00 & 61,25 & 73,50 & 85,75 & 98,00 \\
P7 & 20,00 & 40,00 & 60,00 & 80,00 & 100,00 & 120,00 & 140,00 & 160,00 \\
P8 & 32,25 & 64,50 & 96,75 & 129,00 & 161,25 & 193,50 & 225,75 & 258,00 \\
P9 & 52,13 & 104,25 & 156,38 & 208,50 & 260,63 & 312,75 & 364,88 & 417,00 \\
\hline Total & 135 & 270 & 405 & 540 & 675 & 810 & 945 & $\mathbf{1 0 8 0}$ \\
\hline
\end{tabular}

Para determinar las producciones reales acumuladas $\mathrm{X}_{\mathrm{i}, \mathrm{t}}{ }^{*}$, ajustadas lo máximo posible a sus cuotas $\mathrm{Q}_{\mathrm{i}, \text { }}$, basta con resolver en cada turno un problema del Reparto.

El método de reparto de Alexander Hamilton (1792), conocido también como método de los Restos Mayores, interpreta que los valores $\mathrm{X}_{\mathrm{i}, \mathrm{t}}$ son "lo más parecidos posible" a sus correspondientes cuotas $\mathrm{Q}_{\mathrm{i}, \mathrm{t}}$, cuando la suma de desviaciones absolutas, entre valores y cuotas, elevadas a una potencia $\mathrm{p} \geq 1$, adopta el valor mínimo. Formalmente, a cada turno $\mathrm{t}=1,2, \ldots, \mathrm{T}$, se puede asociar el siguiente programa matemático.

\section{PM-HQ-1:}

$\min F_{t}(X, Q)=\sum_{i=1}^{m}\left|X_{i, t}-Q_{i, t}\right|^{p}, \forall t=1,2, \ldots, T ; p \geq 1$
Sujeto a:

$$
\begin{gathered}
\sum_{i=1}^{m} X_{i, t}=d \times t \\
X_{i, t} \in Z^{+} \cup\{0\}, \quad \forall i=1, \ldots, m
\end{gathered}
$$

En el modelo PM-HQ-1, la función objetivo (2) expresa la minimización de la suma de las desviaciones (potenciadas) entre la producción real acumulada $X_{i, t}$ y su cuota de producción ideal $\mathrm{Q}_{\mathrm{i}, \mathrm{t}}$. La igualdad (3) fuerza el reparto de la capacidad del sistema hasta el t-ésimo turno $(\mathrm{d} \times \mathrm{t})$ entre los elementos del conjunto I. Finalmente, las condiciones (4) establecen la integridad no negativa de las variables $\mathrm{X}_{\mathrm{i}, \mathrm{t}}$.

La solución óptima de PM-HQ-1 ( $\forall \mathrm{t})$ es fácil de obtener iterando el método de los restos mayores tal como se formaliza a continuación.
Algoritmo A1 Método iterado Heijunka de los Restos mayores.
Paso 1: Iniciar el proceso. Hacer $t=1$. Calcular $\lambda_{i}=d_{i} / D, \forall i=1, \ldots, m$.

Paso 2: Calcular las cuotas $Q_{i, t}=\lambda_{i} \times d \times t, \forall i=1, \ldots, m$

Paso 3: Asignar a cada producto la parte entera de su cuota: $X_{i, t}=\left\lfloor Q_{i, t}\right\rfloor(\forall i \in I)$

Paso 4: Determinar los restos de cada tipo de producto: $R_{i, t}=Q_{i, t}-X_{i, t}$, y calcular el resto total o capacidad remanente del sistema productivo: $R_{t}=\sum_{i=1}^{m} R_{i, t}$

Paso 5: Ordenar los restos $R_{i, t}$ de mayor a menor. Sea $L C=\left(i_{1}, \ldots, i_{m}\right)$ la lista de productos ordenados, cumpliendo: $R_{i, t} \geq R_{i^{\prime}, t} \Rightarrow \operatorname{pos}(i, L C)<\operatorname{pos}\left(i^{\prime}, L C\right)$

Paso 6: Repartir la capacidad remanente $R_{t}$ entre los productos con mayor fracción: Hacer $X_{i, t} \leftarrow X_{i, t}+1$ para todo $i \in I$ tal que $\operatorname{pos}(i, L C) \leq R_{t}$.

Paso 7: Test de finalización: $\left\{\begin{array}{c}\text { Si } t=T, \text { Finalizar } \\ \text { Si } t<T \text { Hacer } t \leftarrow t+1 \text {, Ir a Paso } 2\end{array}\right\}$ 
Obviamente, para que un plan Heijunka de producción acumulada sea factible, debe cumplir la Propiedad de Monotonía en Producción: $\mathrm{X}_{\mathrm{i}, \mathrm{t}} \leq \mathrm{X}_{\mathrm{i}, \mathrm{t}+1}(\forall \mathrm{i} \forall \mathrm{t})$. El Algoritmo A1 garantiza dicha propiedad siempre que las cuotas cumplan: $\left[\mathrm{Q}_{\mathrm{i}, \mathrm{t}}\right] \leq\left[\mathrm{Q}_{\mathrm{i}, \mathrm{t}+1}\right\rfloor(\forall \mathrm{i} \forall \mathrm{t})$. Cuando no se cumplen las anteriores condiciones, puede darse la denominada paradoja de Alabama, resultando que la producción acumulada de uno o más artículos en uno o más periodos no es monótona. Para evitar este inconveniente, basta sustituir el Paso 3 del Algoritmo A1 por el Paso 3'. Llamaremos método de los Restos Mayores Modificado $\left(\mathrm{RM}^{\mathrm{M}}\right)$ al que incorpora el Paso 3', constituyendo, así, el Algoritmo A1'.

Paso 3": Asignar a cada producto el máximo entre el suelo de su cuota y la producción acumulada del periodo anterior: $X_{i, t}=\max \left[\left\lfloor Q_{i, t}\right\rfloor, X_{i, t-1}\right](\forall i \forall t)$.

Para fijar ideas, en la Tabla 5, se muestran los resultados de aplicar el Algoritmo A1 al tercer turno de trabajo del Ejemplo 1.

Tabla 5 Plan Heijunka-1: Método de los restos mayores. Reparto de motores (P1 a P9) hasta el turno 3.

\begin{tabular}{c|ccccccccc|c}
\hline Motor & P1 & P2 & P3 & P4 & P5 & P6 & P7 & P8 & P9 & Total \\
\hline$Q_{i, 3}$ & 4,50 & 4,50 & 9,38 & 13,88 & 22,88 & 36,75 & 60,00 & 96,75 & 156,38 & 405 \\
$\left\lfloor Q_{i, 3}\right\rfloor$ & 4 & 4 & 9 & 13 & 22 & 36 & 60 & 96 & 156 & 400 \\
$R_{i, 3}$ & $\mathbf{0 , 5 0}$ & 0,50 & 0,38 & $\mathbf{0 , 8 8}$ & $\mathbf{0 , 8 8}$ & $\mathbf{0 , 7 5}$ & 0,00 & $\mathbf{0 , 7 5}$ & 0,38 & 5 \\
\hline Reparto & 5 & 4 & 9 & 14 & 23 & 37 & 60 & 97 & 156 & $\mathbf{4 0 5}$ \\
\hline
\end{tabular}

Los cálculos son: (i) se asigna la parte entera de la cuota a cada tipo de producto (fila $\left[Q_{i, 3}\right\rfloor$ en Tabla 5), (ii) la suma de valores asignados corresponde a una capacidad utilizada de 400 motores, (iii) se determinan los restos $\mathrm{R}_{\mathrm{i}, 3}$, hasta el turno 3 , para cada producto, resultando una capacidad remanente de 5 motores: $\mathrm{R}_{3}=(405-400)$, y (iv) para saturar la capacidad, se asignan los 5 motores, de uno en uno, según el orden decreciente de los restos (fila $\mathrm{R}_{\mathrm{i}, 3}$ en Tabla 5), siendo los 5 tipos de producto elegidos: P4, P5, P6, P8 y P1.

Las producciones ajustadas $\mathrm{X}_{\mathrm{i}, \mathrm{t}}{ }^{*}$ para el resto de turnos se calculan de forma similar, dando como resultado el plan de producción acumulada que se muestra en la Tabla 6 .
Tabla 6 Heijunka-1: Producción acumulada de motores (P1 a P9) hasta cada turno $(\mathrm{t}=1, \ldots, 8)$.

\begin{tabular}{c|cccccccc}
\hline Plan H.1 & \multicolumn{6}{|c}{ Plan Heijunka de producción acumulada por turnos de trabajo: $X_{i, t}$} \\
\hline Motor/Turno & 1 & 2 & 3 & 4 & 5 & 6 & 7 & 8 \\
\hline P1 & 2 & 3 & 5 & 6 & 8 & 9 & 11 & 12 \\
P2 & 1 & 3 & 4 & 6 & 7 & 9 & 10 & 12 \\
P3 & 3 & 6 & 9 & 13 & 16 & 19 & 22 & 25 \\
P4 & 5 & 9 & 14 & 19 & 23 & 28 & 32 & 37 \\
P5 & 8 & 15 & 23 & 30 & 38 & 46 & 53 & 61 \\
P6 & 12 & 25 & 37 & 49 & 61 & 73 & 86 & 98 \\
P7 & 20 & 40 & 60 & 80 & 100 & 120 & 140 & 160 \\
P8 & 32 & 65 & 97 & 129 & 161 & 193 & 226 & 258 \\
P9 & 52 & 104 & 156 & 208 & 261 & 313 & 365 & 417 \\
\hline Total & 135 & 270 & 405 & 540 & 675 & 810 & 945 & 1080 \\
\hline
\end{tabular}


La transformación del Plan de producción acumulada de la Tabla 6 en el Plan Heijunka-1 de la Tabla 2 es inmediata.

En efecto, dado el turno $\mathrm{t}(\mathrm{t}=1, \ldots, \mathrm{T})$, la producción del producto $\mathrm{i}(\mathrm{i}=1, \ldots, \mathrm{m})$, que notaremos por $\mathrm{x}_{\mathrm{i}, \mathrm{t}}$, se determina restando las producciones acumuladas del producto i hasta los turnos t y t-1. Esto es:

$x_{i, t}=X_{i, t}-X_{i, t-1} \forall i=1, \ldots, m ; \forall t=1, \ldots, T ; X_{i, 0}=0 \forall i=1, \ldots, m$

Nótese que el plan de producción de la Tabla 2 cumple la Propiedad de Homogeneidad en demanda, ya que su concatenación, el número de veces que sea necesario, permite hallar soluciones para planes con horizontes de $\alpha \times \mathrm{T}$ períodos con $\alpha \geq 1$ y entero.
Por tanto, el Plan Heijunka-1 satisface las propiedades: cuota, monotonía en producción y homogeneidad. Además, dicho plan es óptimo para las funciones suma de desviaciones absolutas elevadas a la potencia $p \geq 1$ - ver fórmula (6) -, por no darse, en ninguna de las iteraciones $(1 \leq \mathrm{t} \leq \mathrm{T})$, la paradoja de Alabama cuando aplicamos el Algoritmo A1

$\min S D A^{p}\left(Q_{i, t}\right)=\sum_{t=1}^{T}\left(\sum_{i=1}^{m}\left|X_{i, t}-\lambda_{i} \times d \times t\right|^{p}\right), p \geq 1$

Finalmente, en la Figura 2 se muestra el aspecto gráfico que presentan las producciones acumuladas del Plan Heijunka-1, donde se puede apreciar a simple vista la regularidad de la producción en todos los tipos de producto.
Figura 2 Plan Heijunka-1: Producción acumulada de motores (P1 a P9) hasta cada turno $(\mathrm{t}=1, \ldots, 8)$.

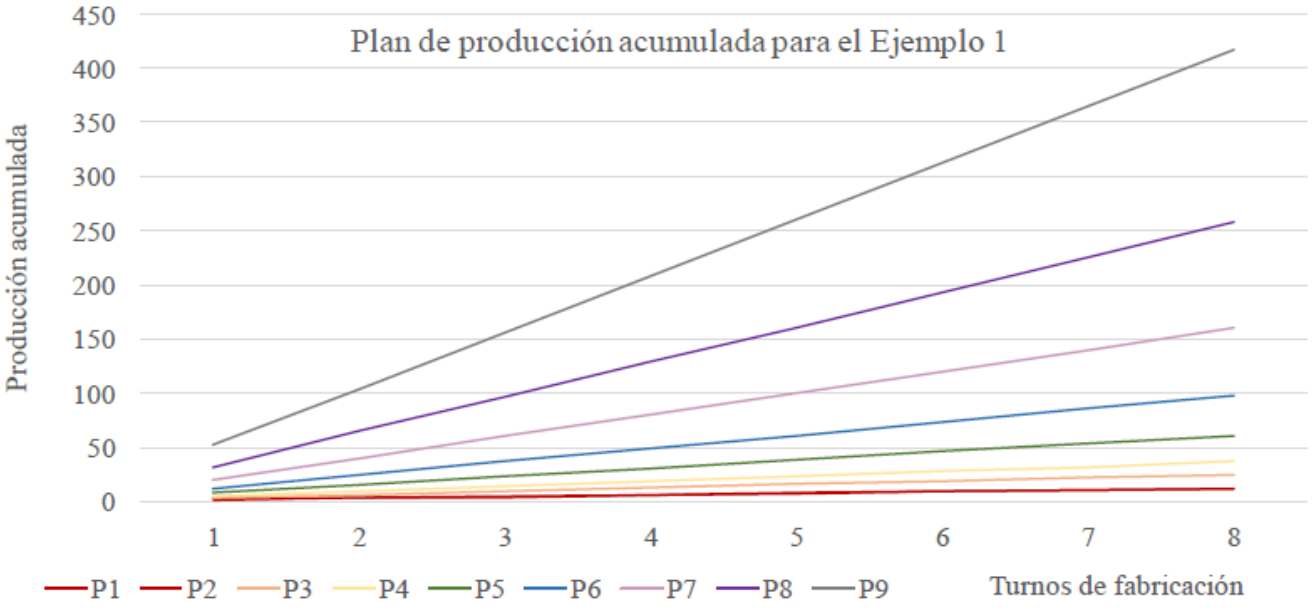

\section{Secuenciación Heijunka}

Heijunka alcanza su punto extremo con las secuencias regulares de producción, de forma que, tras calcular las cantidades a fabricar de cada producto para un periodo, se establece una secuencia de fabricación lo más regular posible. Tal proceso se denomina alisado de la secuencia de producción y al problema, cuando solo afecta a los productos, ignorando otros atributos se denomina Problema de la Variación de las Tasas de Producción (PRV: Product Rate Variation Problem) ([8], [9], [10]).

Por ejemplo, considerando el plan Heijunka-1 de la Tabla 2 , se parte del primer turno y se halla una secuencia de 135 motores de 9 tipos (P1 a P9), teniendo en cuenta un plan de demanda $\vec{d}=(2,1,3,5,8,12,20,32,52)$ y después, se procede de forma similar con los turnos restantes ( 2 al 8). Por tanto, la solución final para dicho plan es una secuencia de fabricación de 1080 motores, compuesta por 8 secuencias parciales de 135 motores cada una, cubriendo así un horizonte temporal de 8 turnos de trabajo.

\subsection{Métodos constructivos basados en Restos Mayores}

Una primera interpretación del concepto "alisado de la secuencia de producción" conduce a funciones objetivo basadas en la suma de desviaciones absolutas elevadas a una potencia $\mathrm{p} \geq 1$ entre las cantidades de cada producto fabricadas hasta un determinado ciclo $t$ y las cantidades ideales que, según las cuotas, deberían estar consolidadas en dicho ciclo. Esta idea se recoge en el programa matemático PM-HQ-2.

$\min S D A^{p}\left(Q_{i, t}\right)=\sum_{t=1}^{T}\left(\sum_{i=1}^{m}\left|\sum_{\tau=1}^{t} x_{i, \tau}-\lambda_{i} t\right|^{p}\right), p \geq 1$

Sujeto a:

$$
\begin{gathered}
\sum_{i=1}^{m} x_{i, t}=1, \forall t=1, \ldots, T \\
\sum_{t=1}^{T} x_{i, t}=d_{i}, \forall i=1, \ldots, m
\end{gathered}
$$




$$
x_{i, t} \in\{0,1\}, \forall i=1, \ldots, m, \forall t=1, \ldots, T
$$

Donde $\mathrm{d}_{\mathrm{i}}$ es la demanda del producto $\mathrm{i}(\forall \mathrm{i} \in \mathrm{I})$, los parámetros $\lambda_{i}$ son las tasas ideales de fabricación de los productos: $\lambda_{\mathrm{i}}=\mathrm{d}_{\mathrm{i}} / \mathrm{T} \quad(\forall \mathrm{i} \in \mathrm{I}), \mathrm{y}_{\mathrm{i}, \mathrm{t}}(\forall \mathrm{i} \forall \mathrm{t})$ es una variable binaria que vale 1 si una unidad del producto $i \in I$ ocupa la t-ésima posición de la secuencia y vale 0 en caso contrario.
Para resolver PM-HQ-2, se puede aplicar el Algoritmo A1 con cuotas para los productos $Q_{i, t}=\lambda_{i} t(\forall i \forall t)$ y las variables de producción acumulada $\mathrm{X}_{\mathrm{i}, \mathrm{t}}=\sum_{\tau=1} \mathrm{t}_{\mathrm{i}, \tau}$. Si al aplicar el Algoritmo A1 no se da la paradoja de Alabama, entonces, la solución es óptima para las funciones objetivo (7), en caso contrario, se puede usar el Algoritmo A2, que garantiza la monotonía en producción, pero no una solución óptima según (7).
Algoritmo A2 Método

constructivo de la secuencia

Heijunka $\pi(\mathrm{T})$ \begin{tabular}{ll}
\hline Paso 1: & Hacer: $t=1, \pi(t)=\{\varnothing\} \forall t,\left(X_{i}=0, \lambda_{i}=d_{i} / D\right) \forall i \in I$. \\
Paso 2: & Crear lista de productos candidatos: $L C(t)=\left\{i \in I: X_{i}<d_{i}\right\}$ \\
Paso 3: & Calcular índices de productos: $f_{i}(t)=\left\{\begin{array}{c}\lambda_{i} t-X_{i}, \forall i \in L C(t) \\
-\infty, \forall i \notin L C(t)\end{array}\right\}$ \\
Paso 4: & Seleccionar el mejor producto: $i^{*}=\operatorname{argmax}\left\{f_{i}(t)\right\}$ \\
Paso 5: & Actualizar: $X_{i^{*}} \leftarrow X_{i^{*}}+1, \pi(t)=\pi(t-1) \cup\left\{i^{*}\right\}$ \\
Paso 6: & Test de finalización: $\left\{\begin{array}{c}\text { Si } t=T, \text { Finalizar } \\
\text { Si } t<T \text { Hacer } t \leftarrow t+1, \text { Ir a Paso } 2\end{array}\right\}$ \\
\hline
\end{tabular}
El Algoritmo A2 es una heurística voraz constructiva que en cada etapa $t$ selecciona un producto candidato con demanda pendiente (Paso 2). Entre los elementos candidatos de la lista $\mathrm{LC}(\mathrm{t})$, se elige el que presenta mayor valor del índice de prioridad $\mathrm{f}_{\mathrm{i}}(\mathrm{t})$ (Pasos 3 y 4). Tras la selección, se actualizan las producciones y se añade el producto elegido $i^{*}$ a la secuencia en fase de construcción (Paso 5). El método finaliza cuando la secuencia $\pi(\mathrm{T})$ ha sido completada hasta el último ciclo T.

En el Algoritmo A2, los índices de prioridad $f_{i}(t)$ son opuestos, respecto a la ordenación de los productos, a los índices asociados a la suma de las desviaciones cuadráticas (i.e. función objetivo (7) con $\mathrm{p}=2$ ). Los índices de desviaciones cuadráticas son:

$$
g_{i}(t)=\sum_{k=1}^{n}\left(X_{k}+\delta_{i, k}-\lambda_{k} t\right)^{2} \quad \forall i \in C L(t)
$$

donde $\mathrm{X}_{\mathrm{k}}$ es el número de productos de tipo $\mathrm{k} \in \mathrm{I}$ que contiene la secuencia consolidada en la etapa previa, $\pi(\mathrm{t}-1)$, y $\delta_{\mathrm{i}, \mathrm{k}}$ es la delta de kronecker: $\delta_{\mathrm{i}, \mathrm{i}}=1 \wedge \delta_{\mathrm{i}, \mathrm{k}}=0 \mathrm{si} \mathrm{i} \neq \mathrm{k}$.

Nótese que el índice $\mathrm{g}_{\mathrm{i}}(\mathrm{t})$ representa la aportación a la función objetivo (7) (en la etapa t) de una unidad de tipo $i \in I$, cuando la secuencia parcial $\pi(t-1)$ contiene $X_{k}$ unidades del producto genérico $\mathrm{k}(\forall \mathrm{k} \in \mathrm{I})$. La equivalencia entre las ordenaciones de los productos según los índices $\mathrm{g}_{\mathrm{i}}(\mathrm{t})$ y $\operatorname{los}$ valores opuestos de $\mathrm{f}_{\mathrm{i}}(\mathrm{t})$ se demuestra a continuación.

\section{TEOREMA 1:}

Dada la secuencia parcial de productos $\pi(\mathrm{t}-1)=\left(\pi_{1}, ., \pi_{\mathrm{t}-1}\right) \mathrm{y}$ una lista de candidatos, $\mathrm{LC}(\mathrm{t})=\left\{\mathrm{i} \in \mathrm{I}: \mathrm{X}_{\mathrm{i}}<\mathrm{d}_{\mathrm{i}}\right\}$, entonces, la ordenación de los productos de LC(t) según los índices $\mathrm{g} .(\mathrm{t})$ es opuesta a la ordenación según los índices $\mathrm{f}_{\mathrm{i}}(\mathrm{t})=\lambda_{\mathrm{i}} \mathrm{t}-\mathrm{X}_{\mathrm{i}}$ $(\forall \mathrm{i} \forall \mathrm{t})$.

\section{DEMOSTRACIÓN:}

En efecto, sea $\mathrm{H}_{\mathrm{k}, \mathrm{t}}=\mathrm{X}_{\mathrm{k}}-\lambda_{\mathrm{k}} \mathrm{t}(\forall \mathrm{k} \in \mathrm{I}, \forall \mathrm{t}=1, \ldots, \mathrm{T})$, entonces, se puede afirmar:

$$
\begin{gathered}
g_{i}(t) \leq g_{j}(t) \Leftrightarrow \sum_{k=1}^{n}\left(X_{k}+\delta_{i, k}-\lambda_{k} t\right)^{2} \leq \sum_{k=1}^{n}\left(X_{k}+\delta_{j, k}-\lambda_{k} t\right)^{2} \Leftrightarrow \\
\sum_{k=1}^{n} \delta_{i, k}^{2}+\sum_{k=1}^{n} H_{k, t}^{2}+2 \sum_{k=1}^{n} \delta_{i, k} H_{k, t} \leq \sum_{k=1}^{n} \delta_{j, k}^{2}+\sum_{k=1}^{n} H_{k, t}^{2}+2 \sum_{k=1}^{n} \delta_{j, k} H_{k, t} \Leftrightarrow \\
\sum_{k=1}^{n} \delta_{i, k} H_{k, t} \leq \sum_{k=1}^{n} \delta_{j, k} H_{k, t} \Leftrightarrow X_{i}-\lambda_{i} t \leq X_{j}-\lambda_{j} t \Leftrightarrow f_{i}(t) \geq f_{j}(t)
\end{gathered}
$$

\section{Ejemplo 2:}

En la línea de modelos mixtos L1 se deben ensamblar, en un turno de trabajo, 120 motores de 3 familias (F1: SUV, F2: VAN y F3: TRUCK). Las demandas planificadas son: 60 motores de F1, 50 de F2 y 10 de F3. Considerando un contexto de producción JIT, determinar una secuencia Heijunka, lo más regular posible, atendiendo a la preservación del mix de producción a lo largo del tiempo.

Considerando la homogeneidad en demanda, el problema consiste en hallar una secuencia cíclica [11] con demandas iguales a 6, 5 y 1 para F1, F2 y F3, respectivamente, y, después, concatenarla 10 veces $(\alpha=10)$ hasta obtener la secuencia de 120 motores. En las tablas 7 y 8, se muestran los resultados de aplicar los algoritmos A1 y A2 al ejemplo. 
Tabla 7 Algoritmo A1: $d \overrightarrow{=}(6,5,1)$. Leyenda: $\left(\lambda_{i} t\right)$ cuota de producción hasta $\mathrm{t},\left(\left[\lambda_{\mathrm{i}} \mathrm{t}\right]\right)$ suelo de la cuota, $\left(\mathrm{R}_{\mathrm{i}, \mathrm{t}}\right)$ resto parcial, $\left(\mathrm{R}_{\mathrm{t}}\right)$ resto a repartir; $\left(\mathrm{X}_{\mathrm{i}, \mathrm{t}}\right)$ producción acumulada; $\left(\pi_{t}\right)$ : Secuencia.

\begin{tabular}{|c|c|c|c|c|c|c|c|c|c|c|c|c|c|c|}
\hline$t$ & \multicolumn{4}{|c|}{$\lambda_{i} t$} & \multicolumn{3}{|c|}{$\left\lfloor\lambda_{i} t\right\rfloor$} & \multicolumn{4}{|c|}{$R_{i, t}$} & \multicolumn{2}{|c|}{$R_{t}$} & \multicolumn{3}{|c|}{$X_{i, t}$} & $\pi_{t}$ \\
\hline Ciclo & 1 & 2 & 3 & 1 & 2 & 3 & 1 & 2 & 3 & Resto & 1 & 2 & 3 & Sec. \\
\hline 1 & 0,50 & 0,42 & 0,08 & 0 & 0 & 0 & $\mathbf{0 , 5 0}$ & 0,42 & 0,08 & 1 & 1 & 0 & 0 & $\mathrm{~F} 1$ \\
\hline 2 & 1,00 & 0,83 & 0,17 & 1 & 0 & 0 & 0,00 & $\mathbf{0 , 8 3}$ & 0,17 & 1 & 1 & 1 & 0 & $\mathrm{~F} 2$ \\
\hline 3 & 1,50 & 1,25 & 0,25 & 1 & 1 & 0 & $\mathbf{0 , 5 0}$ & 0,25 & 0,25 & 1 & 2 & 1 & 0 & $\mathrm{~F} 1$ \\
\hline 4 & 2,00 & 1,67 & 0,33 & 2 & 1 & 0 & 0,00 & $\mathbf{0 , 6 7}$ & 0,33 & 1 & 2 & 2 & 0 & $\mathrm{~F} 2$ \\
\hline 5 & 2,50 & 2,08 & 0,42 & 2 & 2 & 0 & $\mathbf{0 , 5 0}$ & 0,08 & 0,42 & 1 & 3 & 2 & 0 & $\mathrm{~F} 1$ \\
\hline 6 & 3,00 & 2,50 & 0,50 & 3 & 2 & 0 & 0,00 & $\mathbf{0 , 5 0}$ & 0,50 & 1 & 3 & 3 & 0 & $\mathrm{~F} 2$ \\
\hline 7 & 3,50 & 2,92 & 0,58 & 3 & 2 & 0 & 0,50 & $\mathbf{0 , 9 2}$ & $\mathbf{0 , 5 8}$ & 2 & 3 & 3 & 1 & $\mathrm{~F} 3$ \\
\hline 8 & 4,00 & 3,33 & 0,67 & 4 & 3 & 0 & 0,00 & 0,33 & $\mathbf{0 , 6 7}$ & 1 & 4 & 3 & 1 & $\mathrm{~F} 1$ \\
\hline 9 & 4,50 & 3,75 & 0,75 & 4 & 3 & 0 & 0,50 & $\mathbf{0 , 7 5}$ & $\mathbf{0 , 7 5}$ & 2 & 4 & 4 & 1 & $\mathrm{~F} 2$ \\
\hline 10 & 5,00 & 4,17 & 0,83 & 5 & 4 & 0 & 0,00 & 0,17 & $\mathbf{0 , 8 3}$ & 1 & 5 & 4 & 1 & $\mathrm{~F} 1$ \\
\hline 11 & 5,50 & 4,58 & 0,92 & 5 & 4 & 0 & 0,50 & $\mathbf{0 , 5 8}$ & $\mathbf{0 , 9 2}$ & 2 & 5 & 5 & 1 & $\mathrm{~F} 2$ \\
\hline 12 & 6,00 & 5,00 & 1,00 & 6 & 5 & 1 & 0,00 & 0,00 & 0,00 & 0 & 6 & 5 & 1 & $\mathrm{~F} 1$ \\
\hline
\end{tabular}

Atendiendo a la Tabla 7 (Algoritmo A1) y a modo de ejemplo de cálculo, el ciclo $\mathrm{t}=7$ presenta un resto total $\mathrm{R}_{\mathrm{t}}=2$; para obtener el plan $(3,3,1)$, se suma al vector de asignación inicial $(3,2,0)$ el vector $(0,1,1)$, por ser las familias F2 y F3 las que presentan los dos restos mayores $\left(\mathrm{R}_{2,7}=0,92, \mathrm{R}_{3,7}=0,58\right)$.
Tabla 8 Algoritmo A2:

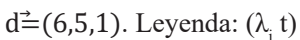
cuota de producción hasta $t$, $\left(\mathrm{X}_{\mathrm{i}, \mathrm{t}-1}\right)$ producción hasta $\mathrm{t}-1$, $\left(\mathrm{f}_{\mathrm{i}}(\mathrm{t})\right)$ índice de prioridad, $\mathrm{X}_{\mathrm{i}, \mathrm{t}}$ producción hasta $\mathrm{t},\left(\pi_{\mathrm{t}}\right)$ secuencia de familias.

\begin{tabular}{|c|c|c|c|c|c|c|c|c|c|c|c|c|c|c|}
\hline$t$ & \multicolumn{4}{|c|}{$\lambda_{i} t$} & \multicolumn{3}{|c|}{$X_{i, t-1}$} & \multicolumn{3}{c|}{$f_{i}(t)=\lambda_{i} t-X_{i, t-1}$} & \multicolumn{3}{|c|}{$X_{i, t}$} & $\pi_{t}$ \\
\hline Ciclo & 1 & 2 & 3 & 1 & 2 & 3 & 1 & 2 & 3 & 1 & 2 & 3 & Sec. \\
\hline 1 & 0,50 & 0,42 & 0,08 & 0 & 0 & 0 & $\mathbf{0 , 5 0}$ & 0,42 & 0,08 & 1 & 0 & 0 & $\mathrm{~F} 1$ \\
\hline 2 & 1,00 & 0,83 & 0,17 & 1 & 0 & 0 & 0,00 & $\mathbf{0 , 8 3}$ & 0,17 & 1 & 1 & 0 & $\mathrm{~F} 2$ \\
\hline 3 & 1,50 & 1,25 & 0,25 & 1 & 1 & 0 & $\mathbf{0 , 5 0}$ & 0,25 & 0,25 & 2 & 1 & 0 & $\mathrm{~F} 1$ \\
\hline 4 & 2,00 & 1,67 & 0,33 & 2 & 1 & 0 & 0,00 & $\mathbf{0 , 6 7}$ & 0,33 & 2 & 2 & 0 & $\mathrm{~F} 2$ \\
\hline 5 & 2,50 & 2,08 & 0,42 & 2 & 2 & 0 & $\mathbf{0 , 5 0}$ & 0,08 & 0,42 & 3 & 2 & 0 & $\mathrm{~F} 1$ \\
\hline 6 & 3,00 & 2,50 & 0,50 & 3 & 2 & 0 & 0,00 & $\mathbf{0 , 5 0}$ & 0,50 & 3 & 3 & 0 & $\mathrm{~F} 2$ \\
\hline 7 & 3,50 & 2,92 & 0,58 & 3 & 3 & 0 & 0,50 & $-0,08$ & $\mathbf{0 , 5 8}$ & 3 & 3 & 1 & $\mathrm{~F} 3$ \\
\hline 8 & 4,00 & 3,33 & 0,67 & 3 & 3 & 1 & $\mathbf{1 , 0 0}$ & 0,33 & $-0,33$ & 4 & 3 & 1 & $\mathrm{~F} 1$ \\
\hline 9 & 4,50 & 3,75 & 0,75 & 4 & 3 & 1 & 0,50 & $\mathbf{0 , 7 5}$ & $-0,25$ & 4 & 4 & 1 & $\mathrm{~F} 2$ \\
\hline 10 & 5,00 & 4,17 & 0,83 & 4 & 4 & 1 & $\mathbf{1 , 0 0}$ & 0,17 & $-0,17$ & 5 & 4 & 1 & $\mathrm{~F} 1$ \\
\hline 11 & 5,50 & 4,58 & 0,92 & 5 & 4 & 1 & 0,50 & $\mathbf{0 , 5 8}$ & $-0,08$ & 5 & 5 & 1 & $\mathrm{~F} 2$ \\
\hline 12 & 6,00 & 5,00 & 1,00 & 5 & 5 & 1 & $\mathbf{1 , 0 0}$ & 0,00 & 0,00 & 6 & 5 & 1 & $\mathrm{~F} 1$ \\
\hline
\end{tabular}


Por su parte, si aplicamos el Algoritmo A2 (Tabla 8), llegados al ciclo $\mathrm{t}=7$, por ejemplo, vemos que la producción consolidada previamente es $\mathrm{X}_{(1,2,3), 6}=(3,3,0)$, y como el mejor valor del índice de prioridad es $f_{3}(7)=0,58$, se selecciona la familia F3.

La secuencia final que ofrecen ambos algoritmos es $\vec{\pi}=(1,2,1,2,1,2,3,1,2,1,2,1)$, siendo óptima para las funciones (7), ya que el Algoritmo A1 garantiza los óptimos en todo ciclo $\mathrm{t}(\mathrm{t}=1, \ldots, 12)$ de las funciones $\sum_{\mathrm{i}=1} \mathrm{~m} \mid$ $\sum_{\tau=1}{ }^{t} X_{i, \tau}-\left.\lambda_{i} t\right|^{p}(p \geq 1)$, cuando no se produce la paradoja de Alabama en ninguno de los 12 ciclos de producción.

\section{Métodos de los Multiplicadores}

Otra forma de cuantificar la regularidad consiste en medir las desviaciones entre los ciclos de entrada (o salida) de los productos en la línea de producción y unos ciclos o fechas ideales de entrada (o salida) que se suceden en el tiempo con periodicidad constante.
Así, se puede considerar que una secuencia es regular si entre cualquier par consecutivo de unidades de un mismo tipo de producto existe una separación, medida en ciclos, lo más similar posible. Por tanto, una secuencia se puede considerar regular si existe una periodicidad en la entrada (o salida) de unidades de cada tipo de producto en la línea de producción, exceptuando, claro está, el caso en que un producto tenga demanda unitaria.

La separación ideal entre unidades de un tipo de producto se determina a partir del plan de demanda $\mathrm{d} \overrightarrow{\mathrm{y}}$ el número total de unidades $T=D$. En efecto, dadas las demandas $d_{i}(\forall$ i) y el horizonte de la secuencia $T$, los períodos $T_{i}(\forall$ i) que debe transcurrir entre la entrada a la línea de dos unidades consecutivas de tipo $\mathrm{i} \in \mathrm{I}$, así como la frecuencia de entrada correspondiente $v_{\mathrm{i}}$ se calculan como sigue.

$$
T_{i}=\frac{T}{d_{i}}=\frac{1}{\lambda_{i}} ; v_{i}=\frac{d_{i}}{T}=\lambda_{i} \quad \forall i \in I
$$

En la Figura 3, se ilustra la separación ideal entre unidades de un mismo tipo de producto, así como una posible localización en la secuencia de las unidades del producto $i \in I$.
Figura 3 Ubicación de unidades del mismo tipo en la secuencia para fechas afines. Separación: $1 / \lambda_{i}$.

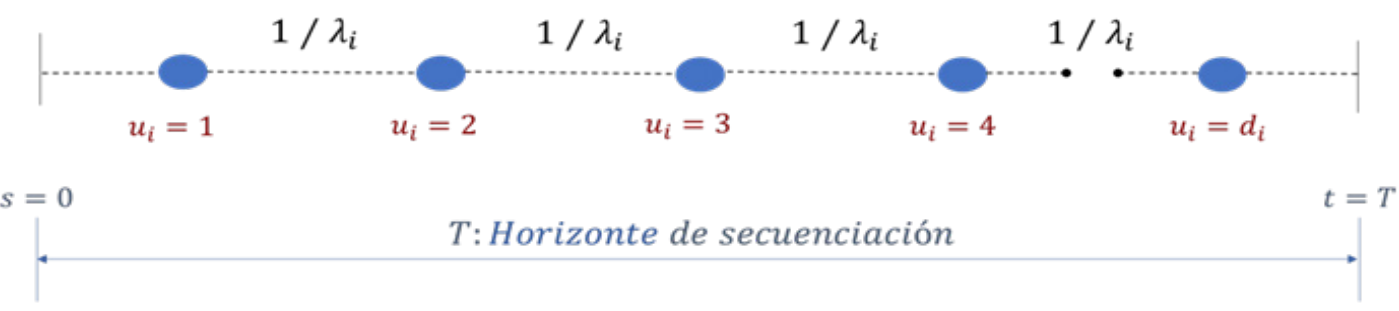

$f_{u_{i}} \equiv u_{i} \times T_{i}=\frac{u_{i}}{\lambda_{i}} \quad \forall i \in I$

Existen múltiples opciones para determinar las fechas idóneas de fabricación; entre dichas opciones son válidas las 5 siguientes:

(1) Fechas mínimas de inicio: corresponden al avance de la producción. Se supone que la primera unidad del producto $\mathrm{i} \in \mathrm{I}$ entra a la línea en el instante 0 (ciclo: $\mathrm{t}=1$ ). Las fechas idóneas de fabricación se determinan según (12):

$f_{u_{i}} \equiv\left(u_{i}-1\right) \times T_{i}=\frac{u_{i}-1}{\lambda_{i}} \quad \forall i \in I$

Donde:

$\mathrm{u}_{\mathrm{i}} \quad$ Número de orden de las unidades de tipo $\mathrm{i} \in \mathrm{I}$ : $\mathrm{u}_{\mathrm{i}}=1, . ., \mathrm{d}_{\mathrm{i}}\left(1^{\mathrm{a}}, 2^{\mathrm{a}}, \ldots\right.$, etc. $)$

$\mathrm{f}_{\mathrm{ui}}$ Fecha de fabricación idónea de la unidad $\mathrm{u}_{\mathrm{i}}(\mathrm{i} \in \mathrm{I})$

(2) Fechas máximas de finalización: corresponden al retraso de la producción. Se supone que la última unidad del producto $i \in I$ sale de la línea de producción en el instante $\mathrm{T}$ (ciclo: $\mathrm{t}=\mathrm{T}$ ). Las fechas idóneas de fabricación se determinan según (13):
(3) Fechas aritméticas de fabricación: corresponden a la media aritmética entre la fecha mínima de inicio y la fecha máxima de finalización para cada valor de $\mathrm{u}_{\mathrm{i}}$. Las fechas idóneas de fabricación se determinan según (14):

$f_{u_{i}} \equiv\left(u_{i}-0,5\right) \times T_{i}=\frac{u_{i}-0,5}{\lambda_{i}} \quad \forall i \in I$

(4) Fechas geométricas de fabricación: corresponden a las medias geométricas entre la fecha mínima de inicio y la fecha máxima de finalización para cada valor de $\mathrm{u}_{\mathrm{i}}$. Las fechas idóneas de fabricación se determinan según (15):

$f_{u_{i}} \equiv \sqrt{u_{i}\left(u_{i}-1\right)} \times T_{i}=\frac{\sqrt{u_{i}\left(u_{i}-1\right)}}{\lambda_{i}} \quad \forall i \in I$

(5) Fechas armónicas de fabricación: corresponden a las medias armónicas entre la fecha mínima de inicio y la fecha máxima de finalización para cada valor de $u_{i}$. Las fechas idóneas de fabricación se determinan según (16): 


$$
f_{u_{i}} \equiv \frac{u_{i}\left(u_{i}-1\right)}{u_{i}-0,5} \times T_{i}=\frac{u_{i}\left(u_{i}-1\right)}{u_{i}-0,5} \times \frac{1}{\lambda_{i}} \forall i \in I
$$

Nótese que las expresiones para el cálculo de fechas idóneas - (12) a (16) - admiten una generalización inmediata.

En efecto, en todas ellas aparecen el valor del mix de producción $\lambda_{\mathrm{i}}$ como divisor, o el período $\mathrm{T}_{\mathrm{i}}$ multiplicando, $\mathrm{y}$, además, un factor multiplicativo, al que notaremos por $b\left(u_{i}\right)$, que depende del número de orden de la unidad $\left(\mathrm{u}_{\mathrm{i}}\right)$.

Por consiguiente, el cálculo de fechas idóneas de fabricación, para el caso de periodicidad por fechas con desplazamiento de unidades, se puede generalizar así:

$$
f_{u_{i}} \equiv b\left(u_{i}\right) \times T_{i}=\frac{b\left(u_{i}\right)}{\lambda_{i}} \quad \forall i \in I
$$

Donde el multiplicador $b\left(u_{i}\right)$ es una función real sobre los números naturales $\mathrm{u}_{\mathrm{i}}\left(1, \ldots, \mathrm{d}_{\mathrm{i}}\right)$ a la que se imponen dos condiciones: (i) monotonía respecto $a u_{i}: b\left(u_{i}-1\right)<b\left(u_{i}\right), y$ (ii) acotación respecto $\mathrm{a}_{\mathrm{i}}: \mathrm{u}_{\mathrm{i}}-1 \leq \mathrm{b}\left(\mathrm{u}_{\mathrm{i}}\right) \leq \mathrm{u}_{\mathrm{i}}$.

Esta generalización da lugar a infinitos métodos de asignación de las fechas idóneas de fabricación, resultando que cada método tiene asociado un criterio multiplicativo que se caracteriza por el multiplicador $b\left(u_{i}\right)$.

Consideremos el Ejemplo 2 para ilustrar la aplicación de las fórmulas (12) a (16), siendo los resultados para las diversas fechas los que se muestran en la Tabla 9.
Tabla 9 Ejemplo 2: Fechas y ciclos ideales de producción $(\mathrm{f}, \mathrm{t})$. Abreviaturas: mínima (min), máxima (max), media aritmética (med), media geométrica (geo) y media armónica (har).

\begin{tabular}{cc|cccccccccc}
\hline$i \in I$ & $u_{i}$ & $f_{\min }$ & $f_{\max }$ & $f_{\text {med }}$ & $f_{\text {geo }}$ & $f_{\text {har }}$ & $t_{\min }$ & $t_{\text {max }}$ & $t_{\text {med }}$ & $t_{\text {geo }}$ & $t_{\text {har }}$ \\
\hline $\mathrm{F} 1$ & 1 & 0,0 & 2,0 & 1,0 & 0,0 & 0,0 & 1 & 2 & 1 & 1 & 1 \\
& 2 & 2,0 & 4,0 & 3,0 & 2,8 & 2,7 & 2 & 4 & 3 & 3 & 3 \\
& 3 & 4,0 & 6,0 & 5,0 & 4,9 & 4,8 & 4 & 6 & 5 & 5 & 5 \\
& 4 & 6,0 & 8,0 & 7,0 & 6,9 & 6,9 & 6 & 8 & 7 & 7 & 7 \\
& 5 & 8,0 & 10,0 & 9,0 & 8,9 & 8,9 & 8 & 10 & 9 & 9 & 9 \\
& 6 & 10,0 & 12,0 & 11,0 & 11,0 & 10,9 & 10 & 12 & 11 & 11 & 11 \\
\hline $\mathrm{F} 2$ & 1 & 0,0 & 2,4 & 1,2 & 0,0 & 0,0 & 1 & 3 & 2 & 1 & 1 \\
& 2 & 2,4 & 4,8 & 3,6 & 3,4 & 3,2 & 3 & 5 & 4 & 4 & 4 \\
& 3 & 4,8 & 7,2 & 6,0 & 5,9 & 5,8 & 5 & 8 & 6 & 6 & 6 \\
& 4 & 7,2 & 9,6 & 8,4 & 8,3 & 8,2 & 8 & 10 & 9 & 9 & 9 \\
& 5 & 9,6 & 12,0 & 10,8 & 10,7 & 10,7 & 10 & 12 & 11 & 11 & 11 \\
\hline $\mathrm{F} 3$ & 1 & 0,0 & 12,0 & 6,0 & 0,0 & 0,0 & 1 & 12 & 6 & 1 & 1 \\
\hline
\end{tabular}

Por otra parte, toda aplicación afín $b\left(u_{i}\right)=u_{i}-\beta_{i}\left(0 \leq \beta_{i} \leq 1\right)$ es un caso particular de la función real $b\left(u_{i}\right)$, lo que permite definir las fechas afines de la forma siguiente:

(6) Fechas afines: corresponden al avance modulado de la producción. Se supone que la primera unidad del producto tipo $i \in I$ entra a la línea en el instante $1-\beta_{\mathrm{i}}$ (primer ciclo: $\mathrm{t}=1$ ). Las fechas idóneas de fabricación se determinan según (18):

$$
f_{u_{i}} \equiv\left(u_{i}-\beta_{i}\right) \times T_{i}=\frac{u_{i}-\beta_{i}}{\lambda_{i}} \quad \forall i \in I\left(0 \leq \beta_{i} \leq 1\right)
$$

Esta definición permite afirmar que las fechas mínimas, máximas y media aritmética son casos partículas de las fechas afines con $\beta_{\mathrm{i}}=1, \beta_{\mathrm{i}}=0$ y $\beta_{\mathrm{i}}=0,5(\forall \mathrm{i})$, respetivamente.

Las fechas idóneas de fabricación dan lugar a una interpretación del PRV distinta a la original, ya que, entonces, puede adaptarse a un problema de programación (scheduling) [12]. En este caso, el problema consiste en programar trabajos en una máquina, asignando una fecha contractual a cada trabajo, con el fin de reducir al mínimo los adelantos y los retrasos entre las fechas reales de fabricación y las fechas idóneas preestablecidas. Un programa matemático representativo del problema es el que sigue 


\section{PM-HF-1:}

$$
\min S D A^{p}\left(f_{u_{i}}\right)=\sum_{i=1}^{m}\left(\sum_{u_{i}=1}^{d_{i}}\left|C_{u_{i}}-f_{u_{i}}\right|^{p}\right), p \geq 1
$$

Sujeto a:

$$
\begin{aligned}
& \sum_{t=1}^{T} t \times x_{u_{i}, t}=C_{u_{i}} \quad \forall u_{i}=1, \ldots, d_{i}, \forall i=1, \ldots, m \\
& C_{u_{i}}<C_{(u+1)_{i}} \forall u_{i}=1, \ldots, d_{i}-1, \forall i=1, \ldots, m \\
& x_{u_{i}, t} \in\{0,1\} \forall u_{i}=1, \ldots, d_{i}, \forall i=1, \ldots, m
\end{aligned}
$$

Donde:

$u_{i} \quad$ Número de orden de las unidades de tipo $i \in I: u_{i}=1, . ., d_{i}$.

$\mathrm{f}_{\mathrm{ui}} \quad$ Fecha de fabricación idónea de la unidad $\mathrm{u}(\mathrm{i} \in \mathrm{I})$

F Conjunto de fechas de fabricación idóneas:

$$
\left\{\mathrm{f}_{\mathrm{ui}}:\left(\mathrm{u}_{\mathrm{i}}=1, \ldots, \mathrm{d}_{\mathrm{i}}\right) \wedge(\mathrm{i} \in \mathrm{I})\right\}
$$

$\mathrm{C}_{\mathrm{ui}}$ Ciclo de fabricación o compleción real de la unidad $\mathrm{u}_{\mathrm{i}}$ $(\mathrm{i} \in \mathrm{I})$

C Conjunto de ciclos de compleción reales:

$$
\left\{\mathrm{C}_{\mathrm{ui}}:\left(\mathrm{u}_{\mathrm{i}}=1, \ldots, \mathrm{d}_{\mathrm{i}}\right) \wedge(\mathrm{i} \in \mathrm{I})\right\}
$$

$\mathrm{x}_{\mathrm{u} \text { i,t }}$ Variable binaria que adopta el valor 1 si la u-ésima unidad de producto de tipo $i \in I$ ocupa la posición $t$ $(\mathrm{t}=1, . ., \mathrm{T})$ de la secuencia, y vale 0 en caso contrario. La relación entre la secuencia $\vec{\pi}$ y las variables $x_{u, t}$ es: $\mathrm{x}_{\mathrm{u}, \mathrm{t}}=1 \Longrightarrow \pi_{\mathrm{t}}=\mathrm{i}, \forall \mathrm{u}_{\mathrm{i}} \forall \mathrm{t}$.

En el modelo PM-HF-1, la función objetivo (19) expresa la minimización de la suma de las desviaciones absolutas, elevadas a $\mathrm{p} \geq 1$, entre los ciclos de compleción real $\left(\mathrm{C}_{\mathrm{u}}\right)$ de las unidades $u_{i}(i \in I)$, y las fechas de fabricación idóneas $\left(f_{u}\right)$ de dichas unidades. Las igualdades (20) sirven para determinar los ciclos de compleción real. Las restricciones (21) fuerzan la monotonía creciente de los ciclos de compleción en función del número de orden de las unidades de cada tipo de producto $i \in I$. Finalmente, las condiciones (22) establecen como binarias las variables $\mathrm{x}_{\mathrm{ui}, \mathrm{t}}$.

En definitiva, el objetivo es que las unidades de cada tipo de producto ocupen posiciones equidistantes en la secuencia, en la medida de lo posible.

Dos casos particulares de (19) son: (i) la minimización de la suma de adelantos y retrasos en las entregas respecto a las fechas establecidas $(\mathrm{p}=1)$, y (ii) la minimización de la suma de dichos adelantos y retrasos elevados al cuadrado $(\mathrm{p}=2)$. Este último caso es propicio para el siguiente teorema.

\section{TEOREMA 2}

Para la función objetivo (19) con p=2, una solución óptima se consigue ordenando las unidades de producto según el orden no decreciente de las fechas de fabricación idóneas $\mathrm{f}_{\text {ui }}$; es decir, ordenando las unidades por fechas según la regla EDD (Earliest Due Date).

\section{DEMOSTRACIÓN:}

En efecto, desarrollando $\operatorname{SDA}^{2}\left(f_{u_{-}}\right)$, se tiene:

$$
\begin{gathered}
\operatorname{SDA}^{2}\left(f_{u_{i}}\right)=\sum_{i=1}^{m}\left(\sum_{u_{i}=1}^{d_{i}}\left|C_{u_{i}}-f_{u_{i}}\right|^{2}\right)= \\
=\sum_{i=1}^{m} \sum_{u_{i}=1}^{d_{i}} C_{u_{i}}^{2}+\sum_{i=1}^{m} \sum_{u_{i}=1}^{d_{i}} f_{u_{i}}^{2}-2 \sum_{i=1}^{m} \sum_{u_{i}=1}^{d_{i}} C_{u_{i}} \times f_{u_{i}}
\end{gathered}
$$

En el desarrollo anterior, la suma de los términos $\mathrm{C}_{\mathrm{ui}}{ }^{2}$ es una constante, correspondiendo a la suma de los cuadrados de los $\mathrm{T}$ primeros números naturales, ya que cada producto $\left(\mathrm{u}_{\mathrm{i}}\right)$ ocupa una (y solo una) posición en la secuencia $\pi \overrightarrow{=}\left(\pi_{1}, \pi_{2}, \ldots\right.$ ,$\left.\pi_{\mathrm{T}}\right)$. Por otra parte, la suma de los cuadrados de las fechas idóneas $\left(\mathrm{f}_{\mathrm{ui}}{ }^{2}\right)$ es una constante. Por tanto, podemos escribir la siguiente equivalencia entre funciones objetivo:

$\min S D A^{2}\left(f_{u_{i}}\right)=\sum_{i=1}^{m} \sum_{u_{i}=1}^{d_{i}}\left|C_{u_{i}}-f_{u_{i}}\right|^{2} \Leftrightarrow \max \Pi\left(f_{u_{i}}\right)=\sum_{i=1}^{m} \sum_{u_{i}=1}^{d_{i}} C_{u_{i}} f_{u_{i}}$

La función objetivo $\Pi\left(\mathrm{f}_{\mathrm{u}}\right)$ corresponde a la suma de productos binarios de los elementos de dos sucesiones numéricas $\left(\mathrm{C}_{\mathrm{ui}} \mathrm{f}_{\mathrm{ui}}\right)$, ambas con valores no negativos, siendo el valor máximo de tal suma-producto el resultante de ordenar las sucesiones en el mismo orden (creciente, en este caso) y multiplicando, posición a posición, sus elementos emparejados.

En resumen, la función objetivo $\max \Pi\left(\mathrm{f}_{\mathrm{ui}}\right)$, equivalente a $\operatorname{minSDA}^{2}\left(f_{u}\right)$, alcanza el óptimo con secuencias $\vec{\pi}=\left(\pi_{1}, \pi_{2}\right.$, $\left.\ldots, \pi_{\mathrm{T}}\right)$ ordenadas por fechas $\mathrm{f}_{\text {ui }}$, según la regla EDD. Así, el programa matemático PM-HF-1 se puede resolver tal como sigue.

(1) Determinar el conjunto de fechas ideales $\mathrm{F}=\left\{\mathrm{f}_{\mathrm{ui}}:\left(\mathrm{u}_{\mathrm{i}}=1, \ldots, \mathrm{d}_{\mathrm{i}}\right) \wedge(\mathrm{i} \in \mathrm{I})\right\}$

(2) Ordenar las fechas ideales del conjunto $\mathrm{F}$ de menor a mayor: Regla EDD.

(3) Secuenciar las unidades $\left(u_{i}=1, \ldots, d_{i}, \forall i \in I\right)$ siguiendo el orden Regla EDD. 
Las propiedades de la regla EDD son extensivas a cualquier conjunto F de fechas idóneas, no obstante, para fijar ideas, nos centraremos en conjuntos de fechas idóneas que adoptan el siguiente formato:

$$
\begin{gathered}
f_{u_{i}} \equiv b\left(u_{i}\right) \times T_{i}=\frac{b\left(u_{i}\right)}{v_{i}}\left(u_{i}=1, \ldots, d_{i}\right) \wedge(i \in I) \\
\text { Cumpliendo: }\left[b\left(u_{i}-1\right)<b\left(u_{i}\right)\right] \wedge\left[u_{i}-1 \leq b\left(u_{i}\right) \leq u_{i}\right] \forall i \in
\end{gathered}
$$

Cada conjunto de fechas (23) da lugar a un método que llamaremos "multiplicador", teniendo en cuenta que los valores $\mathrm{b}\left(\mathrm{u}_{\mathrm{i}}\right)$ actúan como factores multiplicativos de la periodicidad representada por los períodos ideales $T_{i}$.

Entre los infinitos Métodos de los Multiplicadores [13], en la Tabla 10 se muestran los correspondientes a las fechas (12) a (16) y (18).
Tabla 10 Métodos de los Multiplicadores. Período igual a $1 / \lambda_{i}$ (frecuencia: $v_{\mathrm{i}}=\lambda_{\mathrm{i}}$ ). La columna Multiplicador $b\left(u_{i}\right)$ recoge las fórmulas que determinan la sucesión de

\begin{tabular}{|c|c|c|c|}
\hline Nombre & Período $T_{i}$ & $\begin{array}{c}\text { Multiplicador } b\left(u_{i}\right) \\
u_{i}=1, \ldots, d_{i}\end{array}$ & $\begin{array}{c}\text { Sucesión de } \\
\text { multiplicadores }\end{array}$ \\
\hline Fechas mínimas & $T / d_{i}=1 / \lambda_{i}$ & $u_{i}-1$ & $\begin{array}{llllllll}0 & 1 & 2 & 3 & 4 & 5 & \ldots\end{array}$ \\
\hline Fechas armónicas & $T / d_{i}=1 / \lambda_{i}$ & $u_{i}\left(u_{i}-1\right) /\left(u_{i}-0,5\right)$ & $\begin{array}{llll}0 & 1,33 & 2,40 & 3,43\end{array}$ \\
\hline Fechas geométricas & $T / d_{i}=1 / \lambda_{i}$ & $\sqrt{u_{i}\left(u_{i}-1\right)}$ & $\begin{array}{lllll}0 & 1,41 & 2,45 & 3,46\end{array}$ \\
\hline Fechas aritméticas & $T / d_{i}=1 / \lambda_{i}$ & $u_{i}-0,5$ & $\begin{array}{lllll}0,5 & 1,5 & 2,5 & 3,5 & \ldots\end{array}$ \\
\hline Fechas máximas & $T / d_{i}=1 / \lambda_{i}$ & $u_{i}$ & $\begin{array}{lllllll}1 & 2 & 3 & 4 & 5 & \ldots\end{array}$ \\
\hline Fechas afines $\left(\beta_{i}\right)$ & $T / d_{i}=1 / \lambda_{i}$ & $u_{i}-\beta_{i}$ & $1-\beta_{i} 2-\beta_{i} 3-\beta_{i} \ldots$ \\
\hline
\end{tabular}
multiplicadores.
Nótese que los métodos de la Tabla 10 presentan sucesiones de multiplicadores que coinciden, una a una, con las sucesiones de los cinco métodos de los divisores clásicos del problema del reparto de escaños en una cámara de representantes: John Q. Adams (1832), James Dean (1832), Joseph A. Hill (1911), Daniel Webster (1832) y Thomas Jefferson (1794).

La observación anterior permite establecer conexiones interesantes entre el problema PRV y el problema del Reparto ([14], [15]). Por ejemplo, se puede concluir que el objetivo del problema que nos ocupa también admite una representación con las funciones maxmin y minimax que se formulan a continuación.

$$
\max G(\vec{X})=\min _{1 \leq u_{i} \leq d_{i}}\left[\frac{b\left(u_{i}\right)}{d_{i}}\right] \Leftrightarrow \min G^{\prime}(\vec{X})=\max _{1 \leq u_{i} \leq d_{i}}\left[\frac{d_{i}}{b\left(u_{i}\right)}\right]
$$

cumpliendo: $\left[\mathrm{b}\left(\mathrm{u}_{\mathrm{i}}-1\right)<\mathrm{b}\left(\mathrm{u}_{\mathrm{i}}\right)\right] \wedge\left[\mathrm{u}_{\mathrm{i}}-1 \leq \mathrm{b}\left(\mathrm{u}_{\mathrm{i}}\right) \leq \mathrm{u}_{\mathrm{i}}\right](\forall \mathrm{i})$, donde $b\left(u_{i}\right)$ es una función real y las variables $u_{i}(\forall i)$ son enteras positivas.

En cuanto a la resolución del problema, se puede recurrir a cualquier algoritmo de ordenación o, alternativamente, a algoritmos constructivos que conformen la secuencia de fabricación de manera progresiva. En sintonía con la segunda opción, proponemos la siguiente familia de algoritmos:
Algoritmo A3 Métodos de los Multiplicadores
Paso 0 Seleccionar un método. Calcular $T_{i} \forall i \in I$ y seleccionar un multiplicador $b\left(u_{i}\right) \forall i \in I$ (ver detalles en Tabla 10).

Paso 1 Hacer $t=1, u_{i}=1 \forall i \in I, \vec{\pi}=\emptyset$.

Paso 2: Calcular las fechas idóneas de fabricación de las unidades de producto $\left(u_{i}\right)$ con demanda pendiente: $f\left(u_{i}\right)=b\left(u_{i}\right) \times T_{i}=b\left(u_{i}\right) / v_{i}\left(\forall i \in I: u_{i} \leq d_{i}\right)$.

Paso 3: Determinar el tipo de producto $(i \in I)$ con demanda pendiente y con menor fecha idónea de fabricación, sea $i^{*}=\operatorname{argmin}_{\forall u_{i} \leq d_{i}}\left(f\left(u_{i}\right)\right)$.

Paso 4 Actualizar secuencia de fabricación: $\pi_{t}=i^{*}$

Paso 5: Actualizar unidad de producto: $u_{i^{*}}=u_{i^{*}}+1$. Actualizar la fecha idónea en curso: Si $u_{i^{*}} \leq d_{i^{*}}$, Hacer $f\left(u_{i^{*}}\right)=b\left(u_{i^{*}}\right) \times T_{i^{*}}$; Si no, Hacer $f\left(u_{i^{*}}\right) \rightarrow \infty$ (v.gr. $f\left(u_{i^{*}}\right) \rightarrow 10^{10}$ ). Actualizar el ciclo de fabricación: $t=t+1$.

Paso 6 Test de finalización: Si $t<T$, Ir a Paso 3; Si no, Finalizar. 
Las secuencias ofrecidas por el Algoritmo A3 para el Ejemplo 2 se recogen en la Tabla 11, mientras que los mapas de las secuencias distintas se muestran en la Figura
4. Es obvio que las secuencias también se pueden obtener ordenando los productos por fechas idóneas (Tabla 9) según la regla EDD.

Tabla 11 Secuencias regulares obtenidas con 5 métodos multiplicadores para el Ejemplo 2. Métodos con desplazamiento de unidades y período igual a $1 / \lambda_{\mathrm{i}}\left(\right.$ frecuencia: $\left.v_{\mathrm{i}}=\lambda_{\mathrm{i}}\right)$

\begin{tabular}{l|cccccccccccc}
\hline Método|Ciclo & 1 & 2 & 3 & 4 & 5 & 6 & 7 & 8 & 9 & 10 & 11 & 12 \\
\hline Fechas mínimas & F1 & F2 & F3 & F1 & F2 & F1 & F2 & F1 & F2 & F1 & F2 & F1 \\
Fechas armónicas & F1 & F2 & F3 & F1 & F2 & F1 & F2 & F1 & F2 & F1 & F2 & F1 \\
Fechas geométricas & F1 & F2 & F3 & F1 & F2 & F1 & F2 & F1 & F2 & F1 & F2 & F1 \\
Fechas aritméticas & F1 & F2 & F1 & F2 & F1 & F2 & F3 & F1 & F2 & F1 & F2 & F1 \\
Fechas máximas & F1 & F2 & F1 & F2 & F1 & F2 & F1 & F2 & F1 & F1 & F2 & F3 \\
\hline
\end{tabular}

Atendiendo a la Tabla 11, se puede afirmar lo siguiente:

- $\quad$ Las secuencia para las fechas idóneas mínimas $f_{\text {min }}, f_{\text {har }}$ y $f_{\text {geo }}$ es la misma, y en ella se detecta el avance del producto minoritario en el horizonte de secuenciación, dicho producto se lanzará a la línea en uno de los 3 primeros ciclos.

- En la secuencia regular para las fechas idóneas máximas $\left(\mathrm{f}_{\max }\right)$, se produce el retraso de la fabricación del producto minoritario en demanda, ocupando uno de los 3 últimos ciclos en el horizonte de secuenciación.
- En la secuencia regular para fechas idóneas aritméticas $\left(\mathrm{f}_{\text {med }}\right)$, el producto minoritario ocupa una posición intermedia en la secuencia.

Algunas afirmaciones anteriores son extensibles a toda instancia del PRV, sea cual sea el plan de demanda. Por ello, se puede afirmar que el método fechas mínimas favorece a los productos con poca demanda en cuanto al orden de lanzamientos a la línea de producción, mientras que el método fechas máximas favorece a los productos con gran demanda en cuanto a ese mismo aspecto (ver Figura 4).
Figura 4 Mapas de secuencias regulares para el Ejemplo 2.

\begin{tabular}{|c|c|c|c|c|c|c|c|c|c|c|c|c|}
\hline & F1 & F2 & F3 & F1 & F2 & F1 & F2 & F1 & F2 & F1 & F2 & F1 \\
\hline$t$ & 1 & 2 & 3 & 4 & 5 & 6 & 7 & 8 & 9 & 10 & 11 & 12 \\
\hline F1 & & & & & & & & & & & & \\
\hline F2 & & & & & & & & & & & & \\
\hline F3 & & & & & & & & & & & & \\
\hline & & & & & & & & & & & & \\
\hline & F1 & F2 & F1 & F2 & F1 & F2 & F3 & F1 & F2 & F1 & F2 & F1 \\
\hline$t$ & 1 & 2 & 3 & 4 & 5 & 6 & 7 & 8 & 9 & 10 & 11 & 12 \\
\hline F1 & & & & & & & & & & & & \\
\hline F2 & & & & & & & & & & & & \\
\hline F3 & & & & & & & & & & & & \\
\hline & & & & & & & & & & & & \\
\hline & F1 & F2 & F1 & F2 & F1 & F2 & F1 & F2 & F1 & F1 & F2 & F3 \\
\hline$t$ & 1 & 2 & 3 & 4 & 5 & 6 & 7 & 8 & 9 & 10 & 11 & 12 \\
\hline F1 & & & & & & & & & & & & \\
\hline F2 & & & & & & & & & & & & \\
\hline F3 & & & & & & & & & & & & \\
\hline
\end{tabular}


Finalmente, en la Tabla 12 se resume el comportamiento de los métodos expuestos frente a la satisfacción de las propiedades que debe tener una secuencia para que sea considerada regular con plenitud, observando que ninguno de ellos verifica al mismo tiempo las tres propiedades deseables: (i) Cuota inferior y superior, (ii) Monotonía en producción y (iii) Homogeneidad en demanda.

Tabla 12 Verificación de propiedades: (i) Cuota inferior y superior, (ii) Monotonía en producción y (iii) Homogeneidad en demanda, por parte de algunos métodos de secuenciación regular.

\begin{tabular}{lccc}
\hline Método & $\begin{array}{c}\text { Cuota inferior y } \\
\text { superior }\end{array}$ & $\begin{array}{c}\text { Monotonía en } \\
\text { producción }\end{array}$ & $\begin{array}{c}\text { Homogeneidad } \\
\text { en demanda }\end{array}$ \\
\hline Restos mayores & Verifica & No verifica & Verifica \\
Restos mayores $^{\mathrm{M}}$ & Cercano & Verifica & Verifica \\
\hline Fechas mínimas & Superior & Verifica & Verifica \\
Fechas armónicas & No verifica & Verifica & Verifica \\
Fechas geométricas & No verifica & Verifica & Verifica \\
Fechas aritméticas & Cercano & Verifica & Verifica \\
Fechas máximas & Inferior & Verifica & Verifica \\
\hline Fechas afines $(\beta)$ & Función de $\beta$ & Verifica & Verifica \\
\hline
\end{tabular}

\section{Experiencia computacional}

Acto seguido, realizamos una experiencia computacional sobre el conjunto de instancias Nissan-9Eng.I relativas a una planta de motores [16]. Dicho conjunto está compuesto por 23 planes de producción (ver Tabla A1 en Anexo I), siendo sus características principales las que siguen:

- $\quad$ Número de tipos de motor igual a $9(|\mathrm{I}|=9)$.

- Número de estaciones en serie de la línea de fabricación igual a $21(|\mathrm{~K}|=21)$.

- $\quad$ Número de planes de demanda igual a $23(|\mathrm{E}|=23)$.

- Demanda diaria de motores igual a 270 en todos los planes $\left(\mathrm{D}_{\varepsilon}=270(\forall \varepsilon \in \mathrm{E})\right)$.

Los aspectos analizados en el experimento que nos ocupa son los que siguen:

(a) Cálculo de cotas inferiores de la función $\operatorname{SDA}^{2}\left(\lambda_{\mathrm{i}} \mathrm{t}\right)$ para los 23 planes de demanda mediante el método de los restos mayores. Los resultados se recogen en la columna Cota de la Tabla A2 del Anexo II.

(b) Calidad de las soluciones ofrecidas por el método de los restos mayores modificado $\left(\mathrm{RM}^{\mathrm{M}}\right)$ y los métodos de los multiplicadores $\left(\mathrm{f}_{\text {min }}, \mathrm{f}_{\text {har }}, \mathrm{f}_{\text {geo }}, \mathrm{f}_{\text {med }} \mathrm{y} \mathrm{f}_{\text {max }}\right.$ ), respecto a la función $\operatorname{SDA}^{2}\left(\lambda_{\mathrm{i}} \mathrm{t}\right)$. Los resultados se muestran en la Tabla A2 del Anexo II. (c) Porcentaje del número de veces que se produce la paradoja de Alabama, usando el método de los restos mayores y teniendo en cuenta que el número máximo de veces que puede ocurrir la paradoja de Alabama es $|\mathrm{I}| \times(\mathrm{T}-1)$. Los resultados se recogen en la columna $\%$ pA de la Tabla A3 del Anexo III.

(d) Porcentaje del número de veces que cada método viola la propiedad cuota, teniendo en cuenta que el número máximo de violaciones es $|\mathrm{I}| \times \mathrm{T}$. De forma explícita, si se cumple $\left|X_{i, t}-\lambda_{i} t\right| \geq 1$, el método viola la propiedad cuota, en caso contrario, i.e. $\left|\mathrm{X}_{\mathrm{i}, \mathrm{t}}-\lambda_{\mathrm{i}} \mathrm{t}\right|<1(\forall \mathrm{i} \forall \mathrm{t})$, diremos que la secuencia satisface la propiedad cuota. Los resultados se muestran en el bloque $\% \mathrm{v}_{\mathrm{Q}(\varepsilon)}$ de la Tabla A3 del Anexo III.

A modo de resumen, en la Tabla 13 se muestra el desempeño de los procedimientos según los valores medios que adoptan diversas métricas para el conjunto Nissan-9Eng.I. Las 7 métricas seleccionadas son:

$\mathrm{m} 1$. Porcentaje promedio de violaciones de la propiedad cuota inferior (fila $\% \mathrm{v}_{\text {Qinf }}$ ).

$\mathrm{m} 2$. Porcentaje promedio de violaciones de la propiedad cuota superior (fila $\% \mathrm{v}_{\mathrm{Q} \text { sup }}$ ).

m3. Porcentaje promedio de violaciones de la propiedad cuota (fila $\% \mathrm{v}_{\mathrm{Q}}$ ).

m4. Valor medio de la suma de desviaciones cuadráticas (fila $\mathrm{SDA}^{2}$ ). 
m5. Rango medio de la suma de desviaciones cuadráticas (fila $\mathrm{R}\left(\mathrm{SDA}^{2}\right)$ ).

m6. Brecha promedio entre el valor que adopta la función $\operatorname{SDA}^{2}(\lambda, t)$ y la cota inferior para la dicha función (fila GAP).

m7. Rango de la brecha promedio (fila $\mathrm{R}(\mathrm{GAP})$ ).

Atendiendo a los resultados de la Tabla 13, se pueden establecer las sentencias que siguen.

- $\quad$ El método $\mathrm{f}_{\max }$ satisface la propiedad cuota inferior. Este resultado es general, ya que la serie de multiplicadores de $f_{\max }$ es idéntica a la serie de divisores del método de Jefferson (D’Hondt) para el reparto de escaños en una cámara de representantes.
- $\quad$ El método $\mathrm{f}_{\min }$ satisface la propiedad cuota superior. Este resultado es general, ya que los multiplicadores de $\mathrm{f}_{\text {min }}$ coinciden con los divisores del método de Adams.

- $\quad$ El método de los restos mayores modificado $\left(\mathrm{RM}^{\mathrm{M}}\right)$ es el que ofrece mejor desempeño en todas la métricas salvo en la satisfacción de la propiedad cuota inferior.

- $\quad$ Entre los métodos multiplicadores, $\mathrm{f}_{\text {med }}$ es el que tiene mejor desempeño en todas las métricas salvo en las de cuota inferior y superior.

- $\quad$ El método del mejor método (Best) no es mejor que $\mathrm{RM}^{\mathrm{M}}$ en la métrica $\mathrm{R}\left(\mathrm{SDA}^{2}\right)$.
Tabla 13 Valores medios de diversas métricas sobre el desempeño de los métodos $\left[\mathrm{RM}^{\mathrm{M}}, \mathrm{f}_{\text {min }}, \mathrm{f}_{\text {har }}, \mathrm{f}_{\mathrm{geo}}, \mathrm{f}_{\text {med }}\right.$, $f_{\max }$. En la columna Best se recogen los valores medios de los mejores valores para cada métrica $\left[\% \mathrm{v}_{\text {Qinf }}, \% \mathrm{v}_{\text {Qsup, }}\right.$ $\% \mathrm{v}_{\mathrm{Q}}, \mathrm{SDA}^{2}, \mathrm{R}\left(\mathrm{SDA}^{2}\right)$, GAP, $\mathrm{R}(\mathrm{GAP})]$.

\begin{tabular}{c|cccccc|c}
\hline$\varepsilon$ & $R M^{M}$ & $f_{\min }$ & $f_{\text {har }}$ & $f_{\text {geo }}$ & $f_{\text {med }}$ & $f_{\text {max }}$ & Best \\
\hline$\% v_{-} Q_{\text {inf }}$ & $0.10 \%$ & $3.64 \%$ & $0.75 \%$ & $0.62 \%$ & $0.27 \%$ & $\mathbf{0 . 0 0 \%}$ & $0.00 \%$ \\
$\% v_{-} Q_{\text {sup }}$ & $\mathbf{0 . 0 0 \%}$ & $\mathbf{0 . 0 0 \%}$ & $0.15 \%$ & $0.18 \%$ & $0.28 \%$ & $4.24 \%$ & $0.00 \%$ \\
$\% v_{-} Q$ & $\mathbf{0 , 1 0 \%}$ & $3,64 \%$ & $0,90 \%$ & $0,79 \%$ & $0,55 \%$ & $4,24 \%$ & $0,10 \%$ \\
\hline$S D A^{2}$ & $\mathbf{2 7 7 , 8 0}$ & 534,15 & 316,06 & 310,31 & 286,42 & 574,91 & 276,12 \\
$R\left(S D A^{2}\right)$ & $\mathbf{1 5 9 , 3 8}$ & 1160,62 & 253,76 & 221,37 & 159,53 & 1166,22 & 159,53 \\
\hline$G A P$ & $\mathbf{0 , 0 5 8}$ & 1,060 & 0,207 & 0,185 & 0,091 & 1,208 & 0,052 \\
$R(G A P)$ & $\mathbf{0 , 1 9 4}$ & 4,680 & 0,985 & 0,855 & 0,342 & 4,724 & 0,135 \\
\hline
\end{tabular}

el comportamiento de los métodos frente al conjunto de instancias Nissan-9Eng.I; en ellas se aprecia a simple vista las superioridad de los métodos $\mathrm{RM}^{\mathrm{M}}, \mathrm{f}_{\text {med }} \mathrm{y}$ Best.
Figura 5 Diagrama de caja (box plot) relativo al desempeño de los procedimientos para el conjunto de planes Nissan9Eng.I respecto a la función $\operatorname{SDA}^{2}\left(Q_{i, t}\right)$ - ver datos en Tabla A2 del Anexo II-

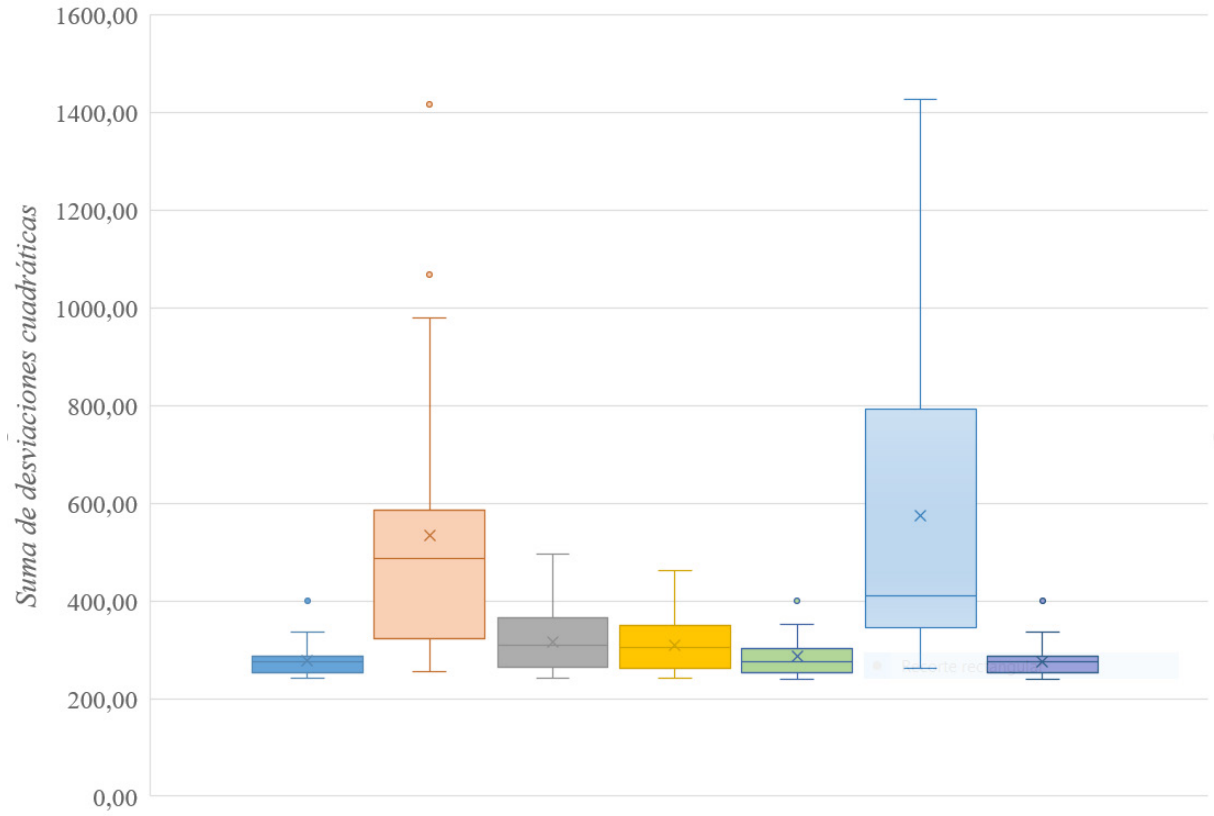

$\square$ - RM-m $\square f$-min $\square f$-har $\square f$-geo $\square f$-med $\square f$-max $\square$ Best 
Figura 6 Diagrama

de caja (box plot)

relativo al desempeño

de procedimientos para

el conjunto de planes

Nissan-9Eng.I respecto a la

propiedad cuota (\%v_Q) -

ver datos en Tabla A3 del

Anexo III.

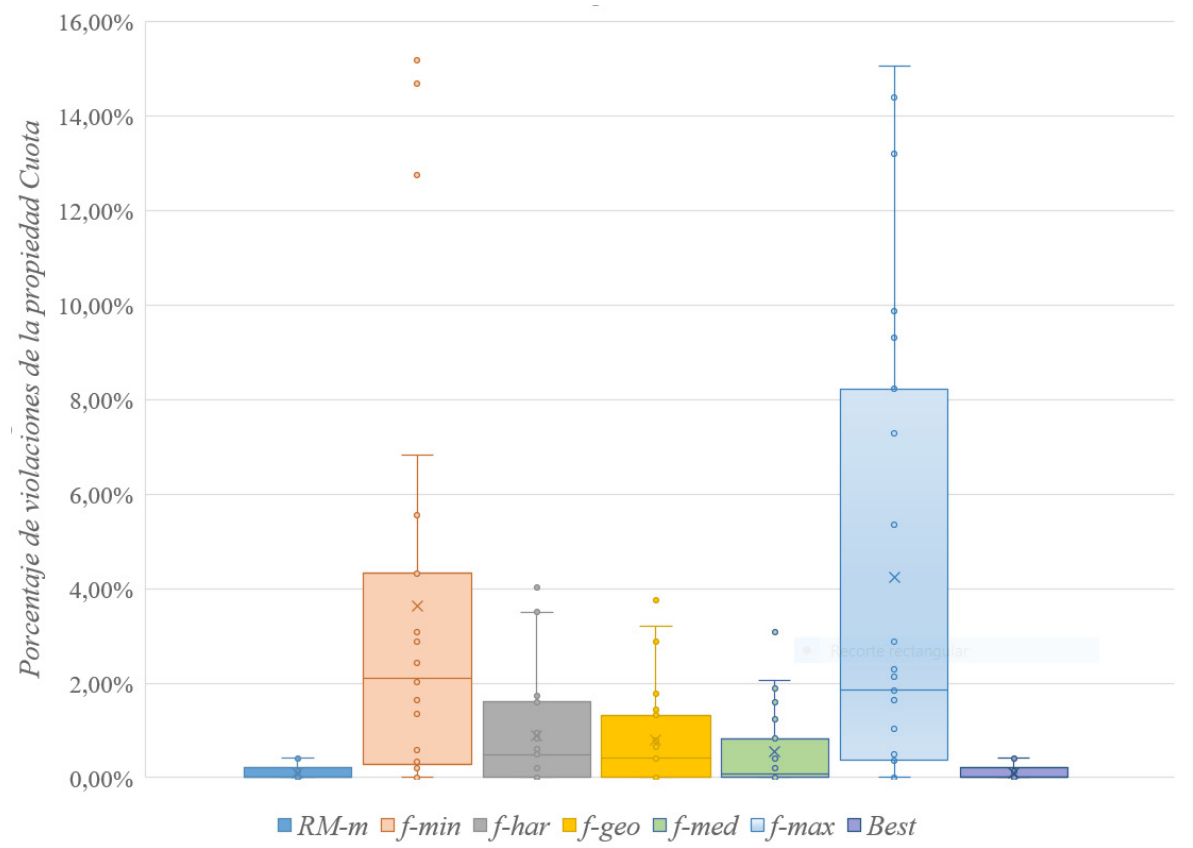

$\square$ RM-m $\square$-min $\square f$-har $\square f$-geo $\square f$-med $\square f$-max $\square$ Best

\section{Conclusiones}

En el presente trabajo se ha definido y justificado un conjunto de infinitos métodos para planificar y secuenciar productos en líneas de montaje de modelos mixtos sujetos al ideario de fabricación Just in time. Hemos denominado a dicho conjunto Métodos de los Multiplicadores.

La definición de los Métodos de los Multiplicadores se justifica a través del Problema del Reparto, cuyas primeras aportaciones formales se dieron en el campo de las Ciencias Sociales, concretamente en el ámbito de la Política con el reparto de escaños de una cámara de representantes, siendo tal problema de gran utilidad, pues se centra en la mejor manera de repartir un recurso escaso medido en unidades indivisibles.

A través del Problema del Reparto, se establece una correspondencia entre los métodos de los divisores (Política) y los métodos de los multiplicadores (Ingeniería), la cual queda justificada por la coincidencia entre series numéricas de ambos conjuntos de métodos.

La fortaleza de esta analogía implícita está en que todas las propiedades de los métodos de los divisores son heredadas automáticamente por los métodos de los multiplicadores y viceversa. Este hecho puede propiciar sinergias en el estudio de ambas problemáticas.

En cuanto a desempeño, el método de los restos mayores modificado, relacionado con el método de Hamilton con monotonía en producción (Cámara en Política), es el que ofrece los mejores resultados frente al conjunto de instancias Nissan-9Eng.I, seguido del método de las fechas aritméticas, cuya imagen en el ámbito político corresponde a los métodos de Webster, Sainte-Laguë y Sainte-Laguë modificado.

\section{Agradecimientos}

Esta investigación ha sido subvencionada por el Ministerio de Ciencia, Innovación y Universidades del Gobierno de España con el proyecto OPTHEUS (ref. PGC2018-095080B-I00), incluyendo Fondos para el desarrollo regional europeo (ERDF: European Regional Development Funds).

\section{Referencias}

[1] Monden Y. (2011) Toyota Production System: An Integrated Approach to Just-In-Time. 4th Edition. New York, Productivity Press.

[2] Hüttmeir A., De Treville S.; Van Ackere, A.; Monnier, L.; Prenninger, J. (2009) Trading off between heijunka and just-in-sequence. International Journal of Production Economics 118 (2): 501-507. https://doi.org/10.1016/j.ijpe.2008.12.014

[3] Yavuz M., Ergin H. (2018) Advanced constraint propagation for the combined car sequencing and level scheduling problem. Computers and Operations Research 100: 128139. https://doi.org/10.1016/j.cor.2018.07.018 
[4] Matzka J., Di Mascolo M., Furmans K. (2012) Buffer sizing of a Heijunka Kanban system. Journal of Intelligent Manufacturing 23: 49-60.

https://doi.org/10.1007/s10845-009-0317-3

[5] Balinski M.L., Young H.P. (1975) The Quota Method of Apportionment. The American Mathematical Monthly 82(7):701-730. Published online: 11 Apr 2018. https://doi.org/10.1080/00029890.1975.11993911

[6] Balinski M.L., Young H.P. (2010) Fair Representation: Meeting the Ideal of One Man, One Vote. Brookings Institution Press, Washington (2010).

[7] Ramírez V., Márquez M.L. (2010) Un Sistema Electoral ecuánime para el Congreso de los Diputados. Revista Española de Ciencia Política 24:139-160.

[8] Miltenburg J.G. (1989) Level schedules for mixed-model assembly lines in just-in-time production systems. Management Science 35(2):192-207. https://doi.org/10.1287/mnsc.35.2.192

[9] Bautista J., Companys R., Corominas A. (1997) Modelling and solving the production rate variation problem (PRVP). Top 5(2): 221-239. https://doi.org/10.1007/BF02568551

[10] Boysen N., Fliedner M., Scholl A. (2009) The product rate variation problem and its relevance in real world mixedmodel assembly lines. European Journal of Operational Research 197(2): 818-824.

https://doi.org/10.1016/j.ejor.2008.06.038
[11] Kubiak W. (2003) Cyclic Just-In-Time Sequences Are Optimal. Journal of Global Optimization 27: 333-347. https://doi.org/10.1023/A:1024847308982

[12] Pinedo M.L. (2016) Scheduling (theory, algorithms, and systems). Springer International Publishing, Berlin. https://doi.org/10.1007/978-3-319-26580-3

[13] Bautista-Valhondo J. (2020) Modelos y Herramientas de Decisión, $1^{\mathrm{a}}$ edición. DEXTRA Editorial, Madrid (2020).

[14] Bautista J., Companys R., Corominas A. (1996) A Note on the Relation between the Product Rate Variation (PRV) Problem and the Apportionment Problem. Journal of the Operational Research Society 47: 1410-1414. https://doi.org/10.2307/3010207

[15] Balinski M., Shahidi N. (1998) A simple approach to the product rate variation problem via axiomatics. Operations Research Letters 22(4-5): 129-135. https://doi.org/10.1016/S0167-6377(98)00008-X

[16] Bautista J., Alfaro R., Batalla C. (2015) Modeling and solving the mixed-model sequencing problem to improve productivity. International Journal of Production Economics 161: 83-95.

http://dx.doi.org/10.1016/j.ijpe.2014.11.018 
Tabla A1. Demandas diarias para cada tipo de motor y plan de demanda $\left(\mathrm{d}_{\mathrm{i}, \varepsilon}\right)$ para las 23 instancias Nissan-9Eng.I ( $\left.\varepsilon \in E\right)$.

\begin{tabular}{c|ccc|cc|cccc|ccc|c}
\hline$\varepsilon \in \mathrm{E}$ & $\mathrm{P} 1$ & $\mathrm{P} 2$ & $\mathrm{P} 3$ & $\mathrm{P} 4$ & $\mathrm{P} 5$ & $\mathrm{P} 6$ & $\mathrm{P} 7$ & $\mathrm{P} 8$ & $\mathrm{P} 9$ & SUV & Van & Truck & Total \\
\hline 1 & 30 & 30 & 30 & 30 & 30 & 30 & 30 & 30 & 30 & 90 & 60 & 120 & 270 \\
\hline 2 & 30 & 30 & 30 & 45 & 45 & 23 & 23 & 22 & 22 & 90 & 90 & 90 & 270 \\
\hline 3 & 10 & 10 & 10 & 60 & 60 & 30 & 30 & 30 & 30 & 30 & 120 & 120 & 270 \\
4 & 40 & 40 & 40 & 15 & 15 & 30 & 30 & 30 & 30 & 120 & 30 & 120 & 270 \\
5 & 40 & 40 & 40 & 60 & 60 & 8 & 8 & 7 & 7 & 120 & 120 & 30 & 270 \\
\hline 6 & 50 & 50 & 50 & 30 & 30 & 15 & 15 & 15 & 15 & 150 & 60 & 60 & 270 \\
7 & 20 & 20 & 20 & 75 & 75 & 15 & 15 & 15 & 15 & 60 & 150 & 60 & 270 \\
8 & 20 & 20 & 20 & 30 & 30 & 38 & 38 & 37 & 37 & 60 & 60 & 150 & 270 \\
\hline 9 & 70 & 70 & 70 & 15 & 15 & 8 & 8 & 7 & 7 & 210 & 30 & 30 & 270 \\
10 & 10 & 10 & 10 & 105 & 105 & 8 & 8 & 7 & 7 & 30 & 210 & 30 & 270 \\
11 & 10 & 10 & 10 & 15 & 15 & 53 & 53 & 52 & 52 & 30 & 30 & 210 & 270 \\
\hline 12 & 24 & 23 & 23 & 45 & 45 & 28 & 28 & 27 & 27 & 70 & 90 & 110 & 270 \\
13 & 37 & 37 & 36 & 35 & 35 & 23 & 23 & 22 & 22 & 110 & 70 & 90 & 270 \\
14 & 37 & 37 & 36 & 45 & 45 & 18 & 18 & 17 & 17 & 110 & 90 & 70 & 270 \\
15 & 24 & 23 & 23 & 55 & 55 & 23 & 23 & 22 & 22 & 70 & 110 & 90 & 270 \\
16 & 30 & 30 & 30 & 35 & 35 & 28 & 28 & 27 & 27 & 90 & 70 & 110 & 270 \\
17 & 30 & 30 & 30 & 55 & 55 & 18 & 18 & 17 & 17 & 90 & 110 & 70 & 270 \\
\hline 18 & 60 & 60 & 60 & 30 & 30 & 8 & 8 & 7 & 7 & 180 & 60 & 30 & 270 \\
19 & 10 & 10 & 10 & 90 & 90 & 15 & 15 & 15 & 15 & 30 & 180 & 60 & 270 \\
20 & 20 & 20 & 20 & 15 & 15 & 45 & 45 & 45 & 45 & 60 & 30 & 180 & 270 \\
21 & 60 & 60 & 60 & 15 & 15 & 15 & 15 & 15 & 15 & 180 & 30 & 60 & 270 \\
22 & 20 & 20 & 20 & 90 & 90 & 8 & 8 & 7 & 7 & 60 & 180 & 30 & 270 \\
23 & 10 & 10 & 10 & 30 & 30 & 45 & 45 & 45 & 45 & 30 & 60 & 180 & 270 \\
\hline & & & & & & & & & & & & & \\
\hline 10
\end{tabular}


Anexo II

Tabla A2. Valores de la función $\operatorname{SDA}^{2}\left(Q_{i, t}\right)$ - fórmula (7) en PM-HQ-2 con p=2 -, para el conjunto de planes Nissan-9Eng.I ( $\varepsilon$ ). Procedimientos: (i) Algoritmo A1 (columna Cota), (ii) Algoritmo A1' o A2 (columna RM ${ }^{\mathrm{M}}$ ) y (iii) Algoritmo A3 (columnas $\mathrm{f}_{\min }, \mathrm{f}_{\text {har }}, \mathrm{f}_{\text {geo }}, \mathrm{f}_{\text {med }}, \mathrm{y} \mathrm{f}_{\text {max }}$ ). Columna Gap*: brecha relativa de la mejor solución respecto a la Cota. Columna $\mathrm{P}^{*}$ : mejor procedimiento.

\begin{tabular}{|c|c|c|c|c|c|c|c|c|c|}
\hline & \multicolumn{7}{|c|}{ Valores de la función $S D A^{2}\left(\lambda_{i} t\right)$} & \multirow[b]{2}{*}{ Gap* } & \multirow[b]{2}{*}{$P^{*}$} \\
\hline & Cota & $R M^{M}$ & $f_{\min }$ & $f_{\text {har }}$ & $f_{\text {geo }}$ & $f_{\text {med }}$ & $f_{\max }$ & & \\
\hline 1 & 400,00 & 000 & & 00,00 & 400,00 & 400,00 & 400,00 & ,000 & $(*)$ \\
\hline 2 & 50,70 & 252,11 & & & 255,05 & 253,38 & & 0,006 & \\
\hline 3 & 263,70 & 287,41 & 514,81 & 319,48 & 320,15 & 299,26 & 574,81 & 0,090 & \\
\hline 4 & & & & & & & & & $f_{\text {med }}$ \\
\hline 5 & 247,92 & 283,62 & 586,53 & 309,76 & 304,57 & 275,42 & & 0,111 & $f_{\text {med }}$ \\
\hline 6 & 262,78 & 274,81 & 386,48 & 288,70 & 278,93 & & & & $R M^{M}$ \\
\hline 7 & 269,17 & 275,46 & 598,43 & 346,43 & 346,76 & 329,54 & 793,43 & 0,023 & \\
\hline 8 & 248,31 & 249,3 & 314,0 & 263 & 261,25 & 250,32 & & & \\
\hline 9 & & 301,20 & & & 348,66 & & & & $f_{\text {med }}$ \\
\hline 10 & 249,35 & 277,99 & 1416,42 & & 462,59 & 334,71 & & 0,115 & $R M^{M}$ \\
\hline 11 & 247,97 & $20<, 12$ & & & 309,21 & & & 0,059 & $R M^{M}$ \\
\hline 12 & 239,93 & 240,72 & 283,51 & 246,99 & 247,01 & 242,04 & & 0,003 & \\
\hline 13 & 239,82 & 240,62 & & & 241, & & & 0,003 & $R M^{M}$ \\
\hline 14 & 237,86 & 24113 & & & 242,05 & 240,47 & & & \\
\hline 15 & 259,96 & 263,05 & 403 & & 273 & 269 & & 0,012 & $R M^{M}$ \\
\hline 16 & 24804 & 248,94 & & & 249,55 & 249,00 & & 0,000 & \\
\hline 17 & 249,91 & 258,14 & 407, & & 266,77 & 263,19 & & 0,033 & $R M^{M}$ \\
\hline 18 & ו & & & & & & & & \\
\hline 19 & 266,48 & 283,52 & 979,81 & 406,04 & 393,15 & 336,48 & 1109,81 & 0,064 & $R M^{M}$ \\
\hline 20 & 268,80 & & & & 306,87 & & & 0,073 & \\
\hline 21 & 312,50 & 336,50 & 487,50 & 370,50 & 361,50 & 352,50 & 892,50 & 0,077 & $R M^{M}$ \\
\hline 22 & 249,76 & 279,4 & 1067,8 & & 378,93 & & & 0,119 & \\
\hline 23 & 267,96 & 297,22 & 547,59 & & 321,59 & 294,26 & & 0,098 & \\
\hline$A v$. & 262.71 & 277.80 & 534.15 & & 310.31 & 286.42 & & 0.052 & $R M^{M}$ \\
\hline
\end{tabular}


Anexo III

Tabla A3. Resultados para el conjunto de planes Nissan-9Eng.I. Columna \%pA: porcentaje del número de veces que aparece la paradoja de Alabama.

Columnas $\left[\mathrm{RM}^{\mathrm{M}}, \mathrm{f}_{\min }, \mathrm{f}_{\mathrm{har}}, \mathrm{f}_{\mathrm{gee}}, \mathrm{f}_{\text {med }}, \mathrm{f}_{\max }\right]$ : porcentaje de insatisfacción de la propiedad cuota, $\% \mathrm{v}_{\mathrm{Q}(\varepsilon)}$, por parte de los 6 procedimientos. Columna Best: porcentaje de violaciones de la propiedad cuota por parte del mejor procedimiento para cada plan.

\begin{tabular}{|c|c|c|c|c|c|c|c|c|}
\hline Planes & Paradoja & \multicolumn{7}{|c|}{$\% v_{-} Q(\varepsilon):$ Porcentaje de violaciones de la propiedad cuota } \\
\hline$\varepsilon$ & $\% p A$ & $R M^{M}$ & $f_{\min }$ & $f_{\text {har }}$ & $f_{\text {geo }}$ & $f_{\text {med }}$ & $f_{\max }$ & Best \\
\hline 1 & $0,00 \%$ & $0,00 \%$ & $0,00 \%$ & $0,00 \%$ & $0,00 \%$ & $0,00 \%$ & $0,00 \%$ & $0,00 \%$ \\
\hline 2 & $0,70 \%$ & $0,00 \%$ & $0,58 \%$ & $0,00 \%$ & $0,00 \%$ & $0,00 \%$ & $0,00 \%$ & $0,00 \%$ \\
\hline 3 & $1,24 \%$ & $0,41 \%$ & $1,65 \%$ & $0,82 \%$ & $0,86 \%$ & $0,82 \%$ & $5,35 \%$ & $0,41 \%$ \\
\hline 4 & $0,41 \%$ & $0,00 \%$ & $0,21 \%$ & $0,00 \%$ & $0,00 \%$ & $0,00 \%$ & $1,03 \%$ & $0,00 \%$ \\
\hline 5 & $2,02 \%$ & $0,12 \%$ & $4,32 \%$ & $0,58 \%$ & $0,41 \%$ & $0,25 \%$ & $2,30 \%$ & $0,12 \%$ \\
\hline 6 & $1,24 \%$ & $0,00 \%$ & $1,36 \%$ & $0,21 \%$ & $0,04 \%$ & $0,00 \%$ & $2,88 \%$ & $0,00 \%$ \\
\hline 7 & $1,86 \%$ & $0,00 \%$ & $5,56 \%$ & $1,73 \%$ & $1,77 \%$ & $1,60 \%$ & $8,23 \%$ & $0,00 \%$ \\
\hline 8 & $0,66 \%$ & $0,00 \%$ & $0,04 \%$ & $0,04 \%$ & $0,04 \%$ & $0,00 \%$ & $0,00 \%$ & $0,00 \%$ \\
\hline 9 & $2,48 \%$ & $0,12 \%$ & $6,83 \%$ & $1,60 \%$ & $1,32 \%$ & $0,41 \%$ & $9,30 \%$ & $0,12 \%$ \\
\hline 10 & $3,26 \%$ & $0,21 \%$ & $15,19 \%$ & $3,50 \%$ & $2,88 \%$ & $1,89 \%$ & $15,06 \%$ & $0,21 \%$ \\
\hline 11 & $1,69 \%$ & $0,04 \%$ & $2,43 \%$ & $0,49 \%$ & $0,45 \%$ & $0,08 \%$ & $2,14 \%$ & $0,04 \%$ \\
\hline 12 & $0,70 \%$ & $0,00 \%$ & $0,29 \%$ & $0,00 \%$ & $0,00 \%$ & $0,00 \%$ & $0,49 \%$ & $0,00 \%$ \\
\hline 13 & $0,41 \%$ & $0,00 \%$ & $0,00 \%$ & $0,00 \%$ & $0,00 \%$ & $0,00 \%$ & $0,04 \%$ & $0,00 \%$ \\
\hline 14 & $0,91 \%$ & $0,00 \%$ & $0,33 \%$ & $0,00 \%$ & $0,00 \%$ & $0,00 \%$ & $0,37 \%$ & $0,00 \%$ \\
\hline 15 & $1,07 \%$ & $0,00 \%$ & $2,02 \%$ & $0,21 \%$ & $0,08 \%$ & $0,08 \%$ & $1,85 \%$ & $0,00 \%$ \\
\hline 16 & $0,04 \%$ & $0,00 \%$ & $0,00 \%$ & $0,00 \%$ & $0,00 \%$ & $0,00 \%$ & $0,00 \%$ & $0,00 \%$ \\
\hline 17 & $1,69 \%$ & $0,00 \%$ & $2,10 \%$ & $0,21 \%$ & $0,08 \%$ & $0,08 \%$ & $1,65 \%$ & $0,00 \%$ \\
\hline 18 & $2,07 \%$ & $0,16 \%$ & $2,88 \%$ & $0,95 \%$ & $0,78 \%$ & $0,49 \%$ & $7,28 \%$ & $0,16 \%$ \\
\hline 19 & $2,48 \%$ & $0,21 \%$ & $12,76 \%$ & $4,03 \%$ & $3,74 \%$ & $3,09 \%$ & $14,40 \%$ & $0,21 \%$ \\
\hline 20 & $1,03 \%$ & $0,21 \%$ & $3,09 \%$ & $0,49 \%$ & $0,45 \%$ & $0,21 \%$ & $1,03 \%$ & $0,21 \%$ \\
\hline 21 & $1,24 \%$ & $0,21 \%$ & $4,32 \%$ & $1,65 \%$ & $1,44 \%$ & $1,23 \%$ & $9,88 \%$ & $0,21 \%$ \\
\hline 22 & $2,68 \%$ & $0,41 \%$ & $14,69 \%$ & $3,50 \%$ & $3,21 \%$ & $2,06 \%$ & $13,21 \%$ & $0,41 \%$ \\
\hline 23 & $1,24 \%$ & $0,21 \%$ & $3,09 \%$ & $0,62 \%$ & $0,66 \%$ & $0,41 \%$ & $1,03 \%$ & $0,21 \%$ \\
\hline$A v$. & $1,35 \%$ & $0,10 \%$ & $3,64 \%$ & $0,90 \%$ & $0,79 \%$ & $0,55 \%$ & $4,24 \%$ & $0,10 \%$ \\
\hline
\end{tabular}

